\title{
Salience and Consumer Choice
}

\section{Citation}

Bordalo, Pedro, Nicola Gennaioli, and Andrei Shleifer. 2013. "Salience and Consumer Choice." Journal of Political Economy 121 (5) (October): 803-843. doi:10.1086/673885.

\section{Published Version}

doi:10.1086/673885

\section{Permanent link}

http://nrs.harvard.edu/urn-3:HUL.InstRepos:27814563

\section{Terms of Use}

This article was downloaded from Harvard University's DASH repository, and is made available under the terms and conditions applicable to Other Posted Material, as set forth at http:// nrs.harvard.edu/urn-3:HUL.InstRepos:dash.current.terms-of-use\#LAA

\section{Share Your Story}

The Harvard community has made this article openly available.

Please share how this access benefits you. Submit a story.

Accessibility 


\title{
Salience and Consumer Choice
}

\section{Pedro Bordalo}

Royal Holloway, University of London

Nicola Gennaioli

Università Bocconi and Innocenzo Gasparini Institute for Economic Research

\section{Andrei Shleifer}

Harvard University

\begin{abstract}
We present a theory of context-dependent choice in which a consumer's attention is drawn to salient attributes of goods, such as quality or price. An attribute is salient for a good when it stands out among the good's attributes relative to that attribute's average level in the choice set (or, more broadly, the choice context). Consumers attach disproportionately high weight to salient attributes, and their choices are tilted toward goods with higher quality/price ratios. The model accounts for a variety of disparate evidence, including decoy effects and contextdependent willingness to pay. It also suggests a novel theory of misleading sales.
\end{abstract}

\section{Introduction}

Imagine yourself in a wine store, choosing a red wine. You are considering a French syrah from the Rhone Valley, selling for $\$ 20$ a bottle, and an Australian shiraz, made from the same grape, selling for $\$ 10$. You

We are grateful to David Bell, Tom Cunningham, Matt Gentzkow, Bengt Holmstrom, Daniel Kahneman, David Laibson, Drazen Prelec, Jan Rivkin, Josh Schwartzstein, Jesse Shapiro, Itamar Simonson, Dmitry Taubinski, Richard Thaler, and four anonymous referees for extremely helpful comments. Shleifer thanks the Kauffman Foundation for research support.

[ Journal of Political Economy, 2013, vol. 121, no. 5]

(C) 2013 by The University of Chicago. All rights reserved. 0022-3808/2013/12105-0004\$10.00 
know and like French syrah better; you think it is perhaps 50 percent better. Yet it sells for twice as much. After some thought, you decide the Australian shiraz is a better bargain and buy a bottle.

A few weeks later, you are at a restaurant, and you see the same two wines on the wine list. Yet both of them are marked up by $\$ 40$, with the French syrah selling for $\$ 60$ a bottle and the Australian shiraz for $\$ 50$. You again think the French wine is 50 percent better, but now it is only 20 percent more expensive. At the restaurant, it is a better deal. You splurge and order the French wine.

This example illustrates what perhaps has happened to many of us, namely, thinking in context and figuring out which of several choices represents a better deal in light of the options we face. In this paper, we try to formalize the intuition behind such thinking. The intuition generalizes what we believe goes through a consumer's mind in the wine example: at the store, the price difference between the cheaper and the more expensive wine is more salient than the quality difference, encouraging the consumer to opt for the cheaper option, whereas at the restaurant, after the markups, the quality difference is more salient, encouraging the consumer to splurge. We argue that this kind of thinking reflects a fundamental feature of decision making, namely, that the consumer's attention is drawn to - and his choice is shaped by - the most salient aspects in the choice context he faces. We present a parsimonious model of salience in decision making for riskless choice and show how it helps account for and unify a broad range of disparate thought experiments, field experiments, and even field data that have been difficult to account for in standard models, and certainly in one model.

Consider a few examples. A car buyer would prefer to pay $\$ 17,500$ for a car equipped with a radio to paying $\$ 17,000$ for a car without a radio but at the same time would not buy a radio separately for $\$ 500$ after agreeing to buy a car for $\$ 17,000$ (Savage 1954). In a related vein, experimental subjects thinking of buying a calculator for $\$ 15$ and a jacket for $\$ 125$ are more likely to agree to travel for 10 minutes to save $\$ 5$ on the calculator than to travel the same 10 minutes to save $\$ 5$ on the jacket (Kahneman and Tversky 1984; Kahneman 2011).

When faced with a choice between a good toaster for $\$ 20$ and a somewhat better one for $\$ 30$, most experimental subjects choose the cheaper toaster. But when a marginally superior toaster is added to the choice set for $\$ 50$, these subjects switch to the middle toaster, violating the axiom of independence of irrelevant alternatives, IIA (Tversky and Simonson 1993).

Imagine sunbathing with a friend on a beach in Mexico. It is hot, and your friend offers to get you an ice-cold Corona from the nearest place, which is 100 yards away. He asks for your reservation price. In the first treatment, the nearest place to buy the beer is a beach resort. In the sec- 
ond treatment, the nearest place is a corner store. Many people would pay more for a beer from a resort than for one from the store, contradicting the fundamental assumption that willingness to pay for a good is independent of context (Thaler 1985, 1999).

When gasoline prices rise, many people switch from higher- to lowergrade gasoline, to an extent that is hard to account for through income effects (Hastings and Shapiro 2013).

Stores often post extremely high regular prices for goods but then immediately put them on sale at substantial discounts. The original prices and percentage discounts are displayed prominently for consumers. In some department stores, more than half the revenues come from sales (Ortmeyer, Quelch, and Salmon 1991).

We suggest that these and several other phenomena can be explained in a unified way using a model of salience in decision making. As described by psychologists Taylor and Thompson $(1982,175)$, "salience refers to the phenomenon that when one's attention is differentially directed to one portion on the environment rather than to others, the information contained in that portion will receive disproportionate weighing in subsequent judgments." Bordalo, Gennaioli, and Shleifer (2012) apply this idea to decisions under risk and present a model in which decision makers overweigh salient lottery states. They find that many anomalies in choice under risk, such as frequent risk-seeking behavior, Allais paradoxes, and preference reversals, obtain naturally when salience influences decision weights. We follow Bordalo et al. in stressing the interplay of attention and choice and extend the concept of salience to riskless choice among goods with different attributes, which may include various aspects of quality but also prices. We then describe decision making by a consumer who overweighs in his choices the most salient attributes of each good he considers, and we show that many of the phenomena just described, as well as several others, obtain naturally in such a model.

In our model, a good's salient attributes are those that stand out or are unusual in the sense of being furthest from those of the "reference good." In the basic version of the model, the reference good is defined as having the average level of each attribute, where the average is taken over the goods in the choice set. The consumer's attention is drawn to the salient attributes, which are then overweighted in his choice. In many situations, salience induces consumers to focus on the relative advantage of goods having a high quality to price ratio. The model thus delivers the fundamental intuition that buyers look for bargains, whether expressed in high quality (relative to price) or low prices (relative to quality).

The salience mechanism generates context effects. The quality/price ratio logic implies that consumers display higher price sensitivity, meaning a steeper trade-off between quality and price, at low price levels. The 
model also provides new insights into the effects of adding to the choice set "decoy" goods with low quality/price ratios. We find that such decoys asymmetrically boost the demand for high-, but not for low-, quality options.

We then extend the model to allow the reference good to also depend on the rationally expected prices of the goods in the choice set. In Thaler's (1985) beer example, the sunbather expects the beer price at the store to be the usual store beer price and the beer price at the resort to be the usual resort beer price. In Hastings and Shapiro's (2013) gasoline example, buyers approach the gas station having in mind rational expectations of gasoline prices. The model predicts strong reactions to unexpected price increases, which make prices more likely to become salient. It also generates a context-dependent willingness to pay through an anchoring-like mechanism. ${ }^{1}$

Context dependence created by salience leads to the central empirical implications of the model. In broad terms, consumers in our model become relatively insensitive to price differences between goods of different qualities when faced with an expected uniform increase in prices. In contrast, consumers are very price sensitive when faced with an unexpected uniform price increase. These central implications are summarized in proposition 5 in Section IV.

Economists have tried several approaches to account for some of the experimental evidence we discuss here: the standard analysis of context effects is information-theoretic (Wernerfelt 1995; Kamenica 2008), while many behavioral models, which we review in Section II.B, emphasize the dependence of choice on external reference points (Kahneman and Tverksy 1979; Simonson and Tversky 1992). The present model offers several advantages. It provides a tractable framework for a fundamental psychological mechanism, based on ex post attention allocation to welldefined salient features of the environment. It accounts for a broad range of context-dependent choices, both in riskless environments and in choice under risk (Bordalo et al. 2012). It provides new insight into how reference points shape valuation and, as a result, can account for evidence that is dumbfounding from the standard perspective, such as Thaler's beer example. More generally, the model's distinctive predictions offer new insights into puzzling evidence in several applications. Finally, the model generates falsifiable predictions, even when some in-

\footnotetext{
${ }^{1}$ Our approach is related to situations in which decision makers evaluate their options using mental accounts (Thaler 1980). Recent research on the interplay of attention and choice includes Mullainathan (2002), Gennaioli and Shleifer (2010), Gabaix (2012), Schwartzstein (2012), and Woodford (2012). The marketing literature also stresses the effect on choice of the set of alternatives that come to the consumer's mind (see Roberts and Lattin 1997).
} 
puts of the salience mechanism (such as quality levels or price expectations) are not fully observable.

The paper is organized as follows. Section II presents the model when context is given by the choice set and establishes the central role of the quality/price ratio in shaping salience and consumer decisions. Section III explores the context effects that arise from manipulations of the choice set, such as changes in price levels and violations of independence of irrelevant alternatives such as decoy and compromise effects. Section IV broadens the notion of context to include expected prices and explores its implications for the demand for quality, including the effects of surprising price changes. Here we show how our model accounts for context-dependent willingness to pay (the Thaler beer example). In Section V, we describe several falsifiable predictions of the model. Section VI presents a new theory of sales showing how discounts can mislead consumers. Section VII presents concluding remarks.

\section{The Model}

A. Setup

A consumer evaluates all $N>1$ goods in a choice set $\mathbf{C} \equiv\left\{\left(q_{k}, p_{k}\right)\right\}_{k=1 \ldots \ldots N}$. Each good $k$ is characterized by its nonnegative quality $q_{k}$ and price $p_{k}$, and we assume, without loss of generality, that price increases in $k$ (i.e., $\left.p_{1}<\cdots<p_{N}\right){ }^{2}$ Quality and price are measured in dollars and known to the consumer. We discuss issues concerning the empirical measurement of quality in Section V. In online Appendix B, we extend the model to the case of goods having multiple quality attributes.

Without salience distortions, a consumer values good $k$ with a linear utility function,

$$
u_{k}=q_{k}-p_{k},
$$

which attaches equal weights to quality and price. A salient thinker departs from (1) by inflating the relative weights attached to the attributes that he perceives to be more salient. As in Bordalo et al. (2012), we say that an attribute (quality or price) is salient for good $k$ in the choice set $\mathbf{C}$ if this attribute "stands out" relative to the good's other attributes. Formally, denote by $(\bar{q}, \bar{p})$ the reference good consisting of average attributes $\bar{q} \equiv \sum_{k} q_{k} / N$ and $\bar{p} \equiv \sum_{k} p_{k} / N$ in $\mathbf{C}$. The salience of quality for a generic good $k$ is then given by $\sigma\left(q_{k}, \bar{q}\right)$, while the salience of price for

\footnotetext{
${ }^{2}$ We think of $\mathbf{C}$ as the set of goods available to the consumer. Unless explicitly mentioned, this set does not include the outside option of not buying, $(0,0)$, which corresponds to zero quality and zero price. Proposition 3 explores the effects of including such an outside option in $\mathbf{C}$.
} 
good $k$ is given by $\sigma\left(p_{k}, \bar{p}\right)$. To define the salience function $\sigma(\cdot, \cdot)$, denote by $a_{k}$ the level of an attribute (quality or price) for good $k$ and by $\bar{a}$ the attribute's average level in $\mathbf{C}$. We then have the following definition.

Definition 1. The salience function $\sigma(\cdot, \cdot)$ is symmetric and continuous and satisfies the following conditions:

1. Ordering. Let $\mu=\operatorname{sgn}\left(a_{k}-\bar{a}\right)$. Then for any $\epsilon, \epsilon^{\prime} \geq 0$ with $\epsilon+\epsilon^{\prime}>0$, we have

$$
\sigma\left(a_{k}+\mu \epsilon, \bar{a}-\mu \epsilon^{\prime}\right)>\sigma\left(a_{k}, \bar{a}\right) .
$$

2. Diminishing sensitivity. For any $a_{k}, \bar{a} \geq 0$ and all $\epsilon>0$, we have

$$
\sigma\left(a_{k}+\epsilon, \bar{a}+\epsilon\right)<\sigma\left(a_{k}, \bar{a}\right) .
$$

We say that, in the choice set $\mathbf{C}$, quality is salient for good $k$ when $\sigma\left(q_{k}, \bar{q}\right)>\sigma\left(p_{k}, \bar{p}\right)$, price is salient for $\operatorname{good} k$ when $\sigma\left(q_{k}, \bar{q}\right)<\sigma\left(p_{k}, \bar{p}\right)$, and price and quality are equally salient when $\sigma\left(q_{k}, \bar{q}\right)=\sigma\left(p_{k}, \bar{p}\right)$.

As we discuss in Bordalo et al. (2012), the properties of definition 1 capture two key features of sensory perception. First, our perceptive apparatus is attuned to detect changes in stimuli. This is captured by ordering, whereby salience increases in contrast: the value $a_{k}$ of an attribute is salient when it is very different from the average value $\bar{a}$ of the same attribute in the choice set. For instance, if a good is much more expensive than average, then its price is very salient. Second, changes in stimuli are perceived with diminishing sensitivity (Weber's law): formally, salience decreases as the value of an attribute uniformly increases for all goods. For instance, the salience of price falls when all prices become uniformly higher. At higher price levels, given price differences are less noticeable.

Ordering and diminishing sensitivity interact in determining salience. Suppose that the price $p_{k}$ of the most expensive good goes up. By ordering, $p_{k}$ becomes more salient. At the same time, the increase in $p_{k}$ increases the average price level $\bar{p}$. By diminishing sensitivity, this reduces the salience of $p_{k}$. When, as in this case, ordering and diminishing sensitivity point in different directions, the trade-off between them is pinned down by the specific salience function adopted. Although most of our results hold under the general definition 1, in what follows we pin down this trade-off by assuming that the salience function is homogeneous of degree zero.

Assumption 1. The salience function satisfies ordering and homogeneity of degree zero, which is defined as $\sigma\left(\alpha \cdot a_{k}, \alpha \cdot \bar{a}\right)=\sigma\left(a_{k}, \bar{a}\right)$ for all $\alpha>0$. 
Together with ordering, homogeneity of degree zero of the salience function implies diminishing sensitivity for positive attribute levels, ${ }^{3}$ so assumption 1 implies definition 1 in this range.

Homogeneity of degree zero characterizes salience in an intuitive way: upon a variation in a good's attribute $a_{k}$, ordering dominates diminishing sensitivity if and only if the change in $a_{k}$ is proportionally larger than the induced change in the average $\bar{a}$. To extend this property to zero attribute levels, we interpret $\sigma\left(a_{k}, 0\right)$ as $\lim _{z \rightarrow 0} \sigma\left(a_{k}, \underline{z}\right)$. Moreover, when comparing $\sigma\left(q_{k}, 0\right)$ and $\sigma\left(p_{k}, 0\right)$, we take the limit with the ratio of the $\underline{z}$ terms constant at 1 . As a consequence, $\sigma\left(q_{k}, 0\right)>\sigma\left(p_{k}, 0\right)$ if and only if $q_{k}>p_{k}$, so ordering is preserved for all nonnegative attribute levels.

An example of a salience function satisfying homogeneity of degree zero, which we previously used in Bordalo et al. (2012), is

$$
\sigma\left(a_{k}, \bar{a}\right)=\frac{\left|a_{k}-\bar{a}\right|}{a_{k}+\bar{a}}
$$

for $a_{k}, \bar{a} \neq 0$, and $\sigma(0,0)=0 .^{4}$

Homogeneity of degree zero highlights the role of a good's quality/ price ratio in determining the salience ranking of its attributes. Take a choice set $\mathbf{C}$ consisting of $N>1$ goods. We then show the following proposition (all proofs are in App. A).

Proposition 1. Let $\left(q_{k}, p_{k}\right)$ be a good that neither dominates nor is dominated by the reference good $(\bar{q}, \bar{p})$, that is, $\left(q_{k}-\bar{q}\right)\left(p_{k}-\bar{p}\right)>0$. The following two statements are equivalent:

1. The higher quality or lower price of $k$ relative to $(\bar{q}, \bar{p})$ is salient iff $q_{k} / p_{k}>\bar{q} / \bar{p}$.

2. Salience is homogeneous of degree zero.

Under assumption 1, salience favors goods with a high quality/price ratio. Proposition 1 captures a central intuition of our model: a good deal is attractive because it draws a consumer's attention to its advantage (high quality or low price) relative to its competitors.

\footnotetext{
${ }^{3}$ To see this, is it sufficient to note that ordering and homogeneity of degree zero imply that salience is an increasing function of the ratio $a_{k} / \bar{a}$ when $a_{k}>\bar{a}$ and of the ratio $\bar{a} / a_{k}$ when $a_{k}<\bar{a}$. Uniform increases in $a_{k}$ and $\bar{a}$ then reduce salience in the two cases. Homogeneity of degree zero is stronger than diminishing sensitivity and, in particular, excludes certain weak forms of the latter. For instance, the salience function $\sigma(x, y)=$ $|x-y| /(x+y+\zeta)$, with $\zeta>0$, satisfies definition 1 but not homogeneity of degree zero (in fact, $\sigma(\alpha x, \alpha y)>\sigma(x, y)$ for $\alpha>1)$.

${ }^{4}$ Because the model is defined for nonnegative attribute levels, the specification of the salience function is slightly different from that in Bordalo et al. (2012): Definition 1 does not include a reflection property (for negative attribute levels), and the denominator in eq. (4) does not feature the absolute value of attributes. The model can be extended in a straightforward way to negative attribute levels.
} 
To complete the model, consider how salience distorts the valuation of a good. Given a salience function $\sigma$, a consumer ranks a good's attributes and distorts their utility weights as follows.

Definition 2. The salient thinker's valuation of good $k$ enhances the relative utility weight attached to the salient attribute (keeping constant the sum of weights attached to quality and price). Formally,

$$
u_{k}^{S}= \begin{cases}\frac{2}{1+\delta} \cdot q_{k}-\frac{2 \delta}{1+\delta} \cdot p_{k} & \text { if } \sigma\left(q_{k}, \bar{q}\right)>\sigma\left(p_{k}, \bar{p}\right) \\ \frac{2 \delta}{\delta+1} \cdot q_{k}-\frac{2}{\delta+1} \cdot p_{k} & \text { if } \sigma\left(q_{k}, \bar{q}\right)<\sigma\left(p_{k}, \bar{p}\right) \\ q_{k}-p_{k} & \text { if } \sigma\left(q_{k}, \bar{q}\right)=\sigma\left(p_{k}, \bar{p}\right),\end{cases}
$$

where $\delta \in(0,1]$ decreases in the severity of salient thinking.

If quality is salient, the relative weight of quality increases, $2 /(1+\delta)$ $>1$, and the relative weight of price decreases, $2 \delta /(1+\delta)<1$, as compared to the rational consumer's valuation. In this case, the salient thinker's price sensitivity is lower than the rational consumer's: a rise $\Delta p$ in price disutility is offset by an increase $\delta \cdot \Delta p$ in quality utility, so that the marginal rate of substitution between a quality increase and a price reduction is $\delta$. If instead price is salient, an increase $\Delta p$ in price is offset by an increase $(1 / \delta) \cdot \Delta p$ in quality, so that the salient thinker's marginal rate of substitution is $1 / \delta$. As $\delta \rightarrow 1$, the salient thinker converges to the rational thinker. As $\delta \rightarrow 0$, the salient thinker considers only the most salient attribute and neglects all others. Normalization of the utility weights ensures that valuation of the good lies between $2 q_{k}$ and $-2 p_{k}$. In definition 2 , utility is assumed to be distorted according to the salience ranking of quality and price. Section II.B discusses the role of this assumption.

To see how the model works, suppose that a consumer is evaluating two bottles of wine, a high-end wine $\left(q_{h}, p_{h}\right)$ and a low-end wine $\left(q_{l}, p_{l}\right)$, where qualities and prices are known and satisfy $q_{h}>q_{l}$ and $p_{h}>p_{l}$. The reference wine has quality $\bar{q}=\left(q_{h}+q_{l}\right) / 2$ and price $\bar{p}=\left(p_{h}+p_{l}\right) / 2$. According to proposition 1 , wine $h$ 's high quality is salient if and only if $q_{h} / p_{h}>\bar{q} / \bar{p}$, which can be written as

$$
\frac{q_{h}}{p_{h}}>\frac{\bar{q}}{\bar{p}}>\frac{q_{l}}{p_{l}} .
$$

Thus, $q_{h}$ is salient for wine $h$ when the high-end wine has a higher quality/price ratio than the low-end wine. Proposition 1 similarly implies that, when condition (6) holds, the lower quality $q_{l}$ is salient for wine $l$. In sum, when the quality/price ratio is higher for the high-quality wine, quality 
is salient for both wines. When the quality/price ratio is higher for the lower-quality, cheaper wine, price is salient for both wines.

Consider how salience affects choice. When prices are salient, namely, when $q_{h} / p_{h}<q_{l} / p_{l}$, expression (5) implies that the low-end wine $l$ is chosen over the high-end wine $h$ provided

$$
\delta \cdot\left(q_{l}-q_{h}\right)-\left(p_{l}-p_{h}\right)>0,
$$

which is easier to satisfy than its rational counterpart, with $\delta=1$. Intuitively, when price is salient, the salient thinker undervalues both wines, but he undervalues the high-end wine more because price is the dimension along which the high-end wine does worse.

Analogously, when $q_{h} / p_{h}>q_{l} / p_{l}$, quality is salient and expression (5) implies that the low-end wine $l$ is chosen over the high-end wine $h$ provided

$$
\left(q_{l}-q_{h}\right)-\delta \cdot\left(p_{l}-p_{h}\right)>0,
$$

which is harder to satisfy than its rational counterpart, with $\delta=1$. Intuitively, when quality is salient, the salient thinker overvalues both wines but overvalues the high-quality wine more because quality is the dimension along which the high-end wine does better.

Salience tilts preferences toward the wine offering the highest quality/price ratio. This is a general property of our model. Suppose that the salient thinker is choosing between $N>1$ goods located along a rational indifference curve, so that each good $k$ provides the same rational utility. Formally, given the quasi-linear utility in (1), a rational indifference curve with utility $u$ is defined as the set of goods $k$ such that $q_{k}-p_{k}=u$. The indifference condition allows us to identify the effect of salience, abstracting from rational utility differences. The $N$ goods display a constant gradient in quality and price, formally, $q_{k}-q_{k^{\prime}}=p_{k}-p_{k^{\prime}}$ for all $k, k^{\prime}=$ $1, \ldots, N$, where $p_{1}<\cdots<p_{N}$. In Appendix A we prove the following proposition.

Proposition 2. Let the choice set $\mathbf{C}$ lie along a rational indifference curve, such that $(\bar{q}, \bar{p})$ is not in $\mathbf{C}$. Then the salient thinker chooses the good with the highest quality/price ratio. Specifically, he chooses good $k^{*}$, where

$$
k^{*}=\arg \max _{k=1, \ldots, N} \frac{q_{k}}{p_{k}}
$$

so that $k^{*}=1$ if $q_{1} / p_{1}>1$, and $k^{*}=N$ if $q_{1} / p_{1}<1$. If $q_{1} / p_{1}=1$, then $q_{1} / p_{1}$ $=q_{k} / p_{k}$ for all $k$ and the consumer is indifferent between any two goods. 
Proposition 2 captures the basic intuition, long recognized in marketing and psychology, that consumers are drawn to goods with a high quality/price ratio (or value per dollar). ${ }^{5}$ It highlights the role of diminishing sensitivity in the salience mechanism, according to which given price differences are less noticeable at higher price levels.

The property that consumers are attracted to goods with high quality/ price ratios extends to more general choice sets, in particular, to the inclusion in $\mathbf{C}$ of the outside option $(0,0)$ of not buying a good. We have the following proposition.

Proposition 3. Let the choice set be $\mathbf{C}=\left\{\left(q_{k}, p_{k}\right)\right\}_{k=1, \ldots, N} \cup\{(0,0)\}$, where goods $k=1, \ldots, N$ lie on a rational indifference curve. Suppose that the cheapest good is preferred to $(0,0)$ if its price is salient, and the most expensive good is preferred to $(0,0)$ if its quality is salient (formally, either $q_{1} / p_{1}>1 / \delta$ or $\left.q_{N} / p_{N} \in(\delta, 1)\right)$. Then the following cases obtain:

i. If $q_{1} / p_{1}<1$, the consumer chooses the most expensive good $\left(q_{N}, p_{N}\right)$, which has the highest quality/price ratio.

ii. If $q_{1} / p_{1}>1$ and price is salient for all goods, the consumer chooses the cheapest good $\left(q_{1}, p_{1}\right)$, which has the highest quality/price ratio. If instead quality is salient for intermediate goods $k$ with $\underline{k} \leq$ $k \leq \bar{k}$, the consumer chooses the highest-quality good, $\bar{k}$, which still has an above-average quality/price ratio.

Adding the outside option does not change our previous result when quality is salient, namely, in case i. Matters change slightly in case ii. While in the absence of the outside option all goods are price salient, adding $(0,0)$ reduces the reference quality and price and that may make intermediate goods quality salient. These intermediate goods may have a lower quality/price ratio than the cheapest good $\left(q_{1}, p_{1}\right)$ but are nonetheless preferred to it because they are seen as a good deal relative to the reference good. Even in this case, however, the chosen good has an aboveaverage quality/price ratio, consistent with the importance of $q / p$ in driving the salient thinker's preferences.

In Appendix B we show how the model works when goods are characterized by several quality dimensions. In this setting, diminishing sensitivity implies that improving one dimension of quality at the expense of another can increase the salience of the weaker dimension. As a consequence, the consumer tends to be attracted toward goods that have sa-

\footnotetext{
${ }^{5}$ The attraction toward goods with a high quality/price ratio (or value per dollar) has been explained by assuming that the consumer experiences a distinct "transaction utility" (Thaler 1999) in that he derives direct pleasure from making a good deal (Jahedi 2011). In our example, the consumer does not derive any special utility from getting a good deal. Instead, a good deal is attractive because it draws the consumer's attention to the dimension in which it does better than its competitor.
} 
lient strengths and yet are balanced in their quality attributes. An uncommonly spacious backseat may enhance consumers' valuation of a car, but not if this comes at the cost of an extremely small trunk. Producers often specialize a little, rarely a lot.

\section{B. Discussion}

The central idea of our model is that consumers focus on-and thus overweight - goods' attributes that stand out in the choice context. This generalizes to riskless settings the logic of salience, initially developed for choice under risk. This approach is also consistent with recent results in neuroeconomics. Hare, Camerer, and Rangel (2009) and Fehr and Rangel (2011) show that subjects evaluate goods by aggregating information about different attributes, with decision weights modulated by attention.

Our model can be easily applied to standard economic problems by introducing salience-based valuation into a "rational" economic model. Doing so requires two key inputs describing the economic problem: (i) the choice set and (ii) the attributes of each good that carry utility. We then add ingredients specific to the salience model, namely, (iii) the reference good, (iv) a salience function, and (v) a specification of salience weighting of attributes. We now discuss these ingredients in turn.

Rational models typically specify the product attributes in the utility function, as well as the choice set faced by the consumer. To apply the salience distortions of definition 2 in our model, the utility function also needs to be separable. ${ }^{6}$ The most straightforward applications of our model feature only two attributes, quality and price, with utility linear in price. ${ }^{7}$ When direct measurement of quality is unavailable, in our approach - as in rational models - a good's quality can be obtained as a latent variable that may depend on the measurable characteristics of the good.

${ }^{6}$ We make three remarks on assuming the separable utility function (1). First, adopting an additive representation of preferences allows us to apply the formalism we developed in Bordalo et al. (2012). Appendix B extends the model to arbitrary weights on quality and price. We have not included the consumer's income $w$ in the numeraire good, from which the consumer obtains utility $w-p_{k}$, because $w$ is not an attribute of the good, so its valuation is not distorted by salience. Second, given separability of the utility function, it is natural to assume linearity in the observed price (measured in dollars) since in most consumer choice settings income effects are not too large. Third, the analysis could be extended to the case of a gain-loss utility, namely, when consumers evaluate the utility of a good's quality and price relative to a reference level.

${ }^{7}$ If, however, firms advertise or specialize along specific dimensions of quality or price, it may be useful to define salience directly on these dimensions, along the lines of the model of App. B. For instance, models of shrouded attributes (Gabaix and Laibson 2006) assume that consumers neglect certain price or quality components. Chetty, Looney, and Kroft (2009) suggest that consumers may not take into account some price dimensions of a purchase such as taxes. In a multiattribute model, the logic of salience may help to explain which prices or quality components endogenously become shrouded (e.g., why consumers neglect them and why firms choose not to compete on them). 
We restrict the admissible salience functions by assuming homogeneity of degree zero, which implies that salience is invariant to scalar transformations of attributes. Under this assumption, we can elicit utility of quality directly within our model from willingness-to-pay experiments; see Section V. While we do not claim that this assumption is universally applicable, it is supported by an emerging paradigm in psychology stressing that people possess an innate "core number system" that compares magnitudes in terms of ratios. ${ }^{8}$ Homogeneity of degree zero is also formally convenient as it ensures that the salience ranking is invariant under linear transformations of the units (dollars) in which the attributes are measured. Thus, even though we defined salience to be a property of dollar attributes, under assumption 1, it can be defined over utils provided that the latter are a scalar transformation of dollars. ${ }^{9}$

We assume that salience weighting is determined by salience ranking, with the magnitude of the salience distortion characterized by the parameter $\delta$. Together with homogeneity of degree zero, this assumption makes our model significantly more tractable and applicable since the effects of salience are characterized in terms of rankings of quality/price ratios. Psychologically, rank-based discounting captures the idea that valuation can be drastically affected by introducing small differences in an attribute such as price (Tverksy 1972; Kim, Novemsky, and Dhar 2012). One feature of rank-based discounting is that valuation can be nonmonotonic, which may be undesirable in some applications. In online Appendix C, we show that with a continuous salience weighting, nonmonotonicities disappear under general conditions and all our results qualitatively carry through.

Finally, an important step in applying our model is to appropriately specify the choice context, or the reference good, with respect to which salience is defined. In deterministic settings, the choice context can be assumed to coincide with the choice set, in line with our formal analysis so far. This is also the case in lab experiments in which subjects are induced to think only about the choice set. ${ }^{10}$

\footnotetext{
${ }^{8}$ According to Feigenson, Dehaene, and Spelke (2004), "To sum up, the findings indicate that infants, children and adults share a common system for quantification." This system exhibits a logarithmic (i.e., ratio-based) representation of numerical magnitude: "numerical representations therefore show two hallmarks: they are ratio-dependent and are robust across multiple modalities of input." Interestingly, the "system becomes integrated with the symbolic number system used by children and adults for enumeration and computation" (309).

${ }^{9}$ Specifically, the ordering and diminishing properties of definition 1 would carry through from dollars to utils, even if utils were an affine transformation of dollars. This is no longer the case if utils are a nonlinear transformation of dollars.

${ }^{10}$ In this paper, we take the choice set as given, but evidence suggests that consumers typically consider only a subset of the options available in the market. The typical number of options in such consideration sets (or evoked sets) ranges from two to five goods (see Hauser and Wernerfelt 1990). This observation justifies our occasional focus on small choice sets. Endogenizing the consideration or evoked set is an important direction of future work; see Hauser and Wernerfelt (1990) and Eliaz and Spiegler (2011).
} 
In stochastic settings, a broader notion of context is needed. In those cases, an attribute's salience also depends on how much this particular realization differs from prior expectations. In Section IV, we extend the model to settings that depend on subjects' expectations (including Thaler's beer example) by assuming that the choice context also includes the agents' rational price expectations. This extension captures the idea that the choice situation brings to the consumer's mind "normal" prices, which then shape the consumer's reference price (Kahneman and Miller 1986).

Several models of consumer choice seek to rationalize context effects by incorporating loss aversion relative to a reference good (see Tversky and Kahneman 1991; Tversky and Simonson 1993; Bodner and Prelec 1994). An implication of these models is a bias toward middle-of-the-road options, which avoid large perceived losses in every attribute. This prediction is hard to reconcile with evidence that in many situations consumers do choose extreme options. Moreover, these models do not speak to the other puzzles reviewed in the introduction, such as the Savage car radio problem, context-dependent willingness to pay, or the Hastings and Shapiro data. ${ }^{11}$

Gabaix (2012) develops a model of rational inattention in which attention to different product attributes is efficiently allocated ex ante, leading the consumer to neglect some of the attributes. In our model, consumer attention to different product attributes is drawn ex post, depending on which attribute stands out.

Models of relative thinking assume that valuation of a good depends on the "referent" levels of its characteristics (Azar 2007; Cunningham 2011). The fundamental assumption is that the marginal utility of a characteristic decreases with the level of its referent, which is reminiscent of the diminishing sensitivity property of salience. Cunningham reproduces some related patterns of choice, such as the Savage car radio puzzle. This approach, however, does not account for patterns of choice in which ordering plays a role, such as the Hastings and Shapiro evidence on gasoline (Sec. IV.A).

Koszegi and Szeidl (2013) build a model that centrally features the idea of ordering: their consumers are essentially salient thinkers who focus on and overweigh those attributes in which options differ the most in terms of utility. Koszegi and Szeidl use their model to shed light on biases in intertemporal choice. By neglecting diminishing sensitivity, their model predicts a strong bias toward concentration; namely, consumers tend to overvalue options whose advantages are concentrated in a single dimension. This bias seems difficult to reconcile with the evidence on

${ }^{11}$ In our model, diminishing sensitivity implies a "loss aversion" type of effect: deviations occurring below the reference attribute level are more salient than those occurring above it. For attributes yielding positive utility, this is reminiscent of the idea that "losses loom larger than gains." The implications for valuation, however, are very different from loss aversion. 
diminishing sensitivity (such as the Savage car radio puzzle) and also with the evident desire of high-quality manufacturers to avoid shortcomings in any aspect of their merchandise.

By combining diminishing sensitivity with ordering within a choice context, our model generates the central prediction linking price sensitivity to context: consumers are relatively insensitive to price differences among goods of different qualities at expected high price levels, while they are price sensitive when faced with unexpected parallel price increases. This mechanism both provides a unified account of several wellknown choice patterns and puzzles and generates new implications.

\section{Salience and Demand for Quality}

We now examine the implications of our model for the reaction of consumers to two distinct manipulations of the choice set. We first explore diminishing sensitivity by considering uniform price shifts of all the goods in the choice set. We then explore ordering by considering changes in the reference good due to the addition of an irrelevant alternative.

\section{A. Price Differences across Contexts and Diminishing Sensitivity}

The wine example from the introduction suggests that a consumer's price sensitivity depends on the price level, namely, that the consumer is more price sensitive when choosing among cheaper goods. This idea is a direct implication of proposition 2 .

Corollary 1. Suppose that the choice set $\mathbf{C}$ lies on a rational indifference curve and that prices are given by $p_{k}+\Delta$ for some constant $\Delta \geq 0$. If for $\Delta=0$ the salient thinker chooses the lowest-quality good $k=1$, then there exists a threshold $\Delta^{*}>0$ such that for $\Delta<\Delta^{*}$ he chooses $k=1$ while for $\Delta>\Delta^{*}$ he chooses the highest-quality good $k=N$.

The corollary establishes a sense in which the demand for quality is increasing in the level of prices. ${ }^{12}$ In the wine example from the introduction, the consumer chooses between two wines of qualities $q_{h}=30$ and $q_{l}=20$. At the store, prices are $p_{h}=20$ and $p_{l}=10$. At the restaurant, prices are uniformly increased by $\Delta p=40$. Though the quality gradient $q_{h}-q_{l}$ and the price gradient $p_{h}-p_{l}$ are the same in the two situations, the consumer chooses the cheap wine at the store and the expensive wine at the restaurant. Owing to diminishing sensitivity of the salience function, the price difference of 10 between the wines is more noticeable to the consumer at the low price level of the store than at the

\footnotetext{
${ }^{12}$ For a rational thinker with $\delta=1$, demand is independent of the price shift $\Delta$.
} 
high price level of the restaurant. To see how this example illustrates the logic of corollary 1 , note that at the store, the cheaper wine has a higher quality/price ratio $(20 / 10>30 / 20)$, while at the restaurant the ranking is reversed $(20 / 50<30 / 60)$. Price is salient at the store; quality is salient at the restaurant.

Diminishing sensitivity can lead to several of the preference reversals discussed in the introduction, such as Savage's (1954) car radio problem or Kahneman and Tversky's (1984) jacket-calculator problem, formalizing Thaler's (1980) intuitive argument based on Weber's law. ${ }^{13}$ Owing to diminishing sensitivity, the salience mechanism induces consumers to display higher price sensitivity for choice among cheaper goods. ${ }^{14}$ As we show in Section IV, in nondeterministic settings the strength of this effect depends on price expectations.

\section{B. Decoy and Compromise Effects}

A well-documented anomaly in both marketing and psychology is the socalled decoy effect (Huber, Payne, and Puto 1982; Tversky and Simonson 1993): adding to a pairwise choice an option dominated by one of the goods boosts the demand for the dominating good. Another well-known anomaly is the compromise effect (Simonson 1989): adding an extreme option to a pairwise choice induces subjects to change their preferences toward the middle-of-the-road, or compromise, option. Assuming, along the lines of Section II, that the consumer is perfectly informed about the available goods, both anomalies constitute violations of the IIA. ${ }^{15}$

Our model can provide an intuitive account for these phenomena as a consequence of the impact of the added option on salience. The proposition below describes conditions under which such preference reversals arise, providing a novel and testable prediction of our model.

\footnotetext{
${ }^{13}$ A salient thinker is more likely to buy a car radio when the price of the radio is added to the price of the car than when the radio is sold in isolation, separately from the car purchase. To see how this works, note that diminishing sensitivity implies that the salience of the price $p_{r}$ of the radio is higher when evaluated in isolation, $\sigma\left(p_{r}, p_{r} / 2\right)$, than against the backdrop of the much higher price $p$ of the car, $\sigma\left(p+p_{r}, p+p_{r} / 2\right)$. Intuitively, the cost of the add-on is less salient when it is "hidden" behind the high price of the core good.

${ }_{14}$ This mechanism differs from models based on loss aversion. In Bodner and Prelec's (1994) model, consumers evaluate each good's gains and losses relative to the same reference good, namely, the "centroid" (or average) good in the choice set. As prices increase uniformly, the gains/losses relative to the reference price stay constant, leaving choice unchanged. In our model, in contrast, as prices increase, a given price difference becomes less salient because salience is evaluated relative to not experiencing an attribute and not with respect to experiencing its reference level. Price levels can also affect the rational consumer's choice through income effects, but in the opposite direction of our prediction: under concave utility, consumers are more price sensitive at higher price levels.

${ }^{15}$ Wernerfelt (1995) and Kamenica (2008) explain the decoy effects by suggesting that decoys indirectly provide consumers with information about the quality of the products.
} 
Proposition 4. Let $\mathbf{C}=\left\{\left(q_{l}, p_{l}\right),\left(q_{h}, p_{h}\right)\right\}$ and assume that $\left(q_{h}, p_{h}\right)$ is preferred to $\left(q_{l}, p_{l}\right)$ if and only if its higher quality is salient; formally,

$$
-(1-\delta)\left(p_{h}-p_{l}\right) \leq \Delta u \leq(1-\delta)\left(q_{h}-q_{l}\right),
$$

where $\Delta u=\left(q_{h}-q_{l}\right)-\left(p_{h}-p_{l}\right)$ is the rational utility difference between the goods. Let $\left(q_{d}, p_{d}\right)$ be a decoy good such that $\left(q_{h}, p_{h}\right)$ retains aboveaverage quality and price in the enlarged choice set $\mathbf{C}_{d}=\mathbf{C} \cup\left\{\left(q_{d}, p_{d}\right)\right\}$ (i.e., $q_{h}>\bar{q}$ and $p_{h}>\bar{p}$ ). Then the following cases obtain:

i. If $q_{l} / p_{l}>q_{h} / p_{h}$, so that price is salient and $\left(q_{l}, p_{l}\right)$ is chosen from $\mathbf{C}$, then for any $\left(q_{d}, p_{d}\right)$ satisfying

$$
\frac{q_{d}}{p_{d}}<\frac{q_{h}}{p_{h}}+\frac{p_{l}}{p_{d}}\left(\frac{q_{h}}{p_{h}}-\frac{q_{l}}{p_{l}}\right),
$$

$\operatorname{good}\left(q_{h}, p_{h}\right)$ is quality salient in $\mathbf{C}_{d}$. Moreover, there exist decoys satisfying the previous condition and $q_{d}>q_{h}, p_{d}>p_{h}$ such that $\left(q_{h}, p_{h}\right)$ is chosen from $\mathbf{C}_{d}$.

ii. If $q_{l} / p_{l}<q_{h} / p_{h}$, so that quality is salient and $\left(q_{h}, p_{h}\right)$ is chosen from C, then there exists no decoy such that $q_{d} / p_{d} \leq q_{l} / p_{l}$ and $\left(q_{h}, p_{h}\right)$ is price salient in $\mathbf{C}_{d}$. In particular, for no decoy satisfying $q_{d} / p_{d}$ $\leq q_{l} / p_{l}$ is $\left(q_{l}, p_{l}\right)$ chosen from $\mathbf{C}_{d}$.

Result i says that the decoy must be a bad deal, namely, a good with a significantly lower quality/price ratio than other available goods. When $q_{d} / p_{d}$ is low, it lowers the quality/price ratio of the reference good to the point that $q_{h} / p_{h}>\bar{q} / \bar{p}$. As a consequence, the quality of $\left(q_{h}, p_{h}\right)$ becomes salient, so this good is now overvalued and is chosen in the enlarged choice set (provided that the decoy is not so good that the consumer prefers it to $\left.\left(q_{h}, p_{h}\right)\right)$.

To illustrate this mechanism, consider again the wine example from the introduction, with a variation in which a third, more expensive and high-quality wine $\left(q_{d}=30, p_{d}=30\right)$ is added to the wine selection at the store:

$$
\begin{aligned}
\mathbf{C}^{\text {store }} & =\{(30,20),(20,10)\}, \\
\mathbf{C}^{\text {store }(d)} & =\{(30,20),(20,10),(30,30)\} .
\end{aligned}
$$

The decoy wine $(30,30)$ yields lower utility than the original options, as

$$
u(30,30)=0<u(30,20)=u(20,10)=10 .
$$


For a rational decision maker, the inclusion of the decoy in the choice set is irrelevant.

Consider the choice of a salient thinker. As shown in Section III.A, in $\mathbf{C}^{\text {store }}$ the salient thinker picks the low-end wine $\left(q_{l}, p_{l}\right)$ because $\left(q_{h}, p_{h}\right)$ has a below-average quality/price ratio, so that its high price is salient. When the decoy is added, however, things change. In fact, the quality/ price ratio of the decoy wine, $30 / 30$, is lower than the quality/price ratio of the high-end wine, $30 / 20$. Thus, by comparison with $\left(q_{d}, p_{d}\right)$, the highend wine $\left(q_{h}, p_{h}\right)$ seems a better deal than in the original choice set! Formally, in $\mathbf{C}^{\text {store }(d)}$, the reference wine is $(26.7,20)$. The high-end wine delivers above reference quality $30>26.7$ at the reference price 20. As a consequence, the quality of $\left(q_{h}, p_{h}\right)$ becomes salient, which implies that $\left(q_{h}, p_{h}\right)$ is now preferred to $\left(q_{l}, p_{l}\right)$, yielding the decoy effect.

In this example, the decoy is dominated by $\left(q_{h}, p_{h}\right)$. Critically, however, case i above shows that this violation of IIA can also arise when $q_{d}>q_{h}$ and $p_{d}>p_{h}$ so that, in the augmented choice set, the high-end wine provides intermediate levels of quality and price but offers a good quality/ price ratio when compared to the decoy. This creates a compromise effect in our model, with the same logic as the decoy effect.

Consider next result ii of proposition 4. It says that the decoy effect is asymmetric in the sense that it does not reverse an initial preference for high-quality goods. When quality is salient in pairwise choice (namely, $\left.q_{h} / p_{h}>q_{l} / p_{l}\right)$, adding a decoy to the lower-quality good $\left(q_{l}, p_{l}\right)$ may cause its low price to become salient. However, since the decoy reduces the quality/price ratio of the reference good, it cannot at the same time make the high price of $\left(q_{h}, p_{h}\right)$ salient. Since this high-end good remains quality salient, it is still chosen in the enlarged choice set. There are instances, not contemplated in proposition 4 , in which a decoy may increase the relative valuation of a lower-quality good. ${ }^{16}$ However, proposition 4 captures an important asymmetry generated by our model whereby goods with high quality and high price are more likely to benefit from decoys than their low-quality, low-price competitors.

The asymmetry of decoy effects is consistent with Heath and Chatterjee's (1995) survey of experimental and field results on decoys. In agreement with our predictions, the authors document a robust asymmetry in the workings of the decoy effect: adding appropriate decoys typically boosts experimental subjects' demand for high-quality goods at the expense of demand for low-quality goods. In contrast, adding decoys for low-quality goods does not boost the demand for these goods. In this respect, our model differs substantially from formalizations of context

${ }^{16}$ These include decoys with extremely high quality/price ratios but very low levels of quality. 
dependence based on loss aversion (Tversky and Simonson 1993; Bodner and Prelec 1994), where consumers minimize losses across all attributes and mechanically prefer middle-of-the-road options, so that asymmetries do not arise.

\section{Salience and Expectations}

So far we took a narrow view of the choice context by identifying it with the choice set $\mathbf{C}$. A long tradition in psychology, however, stresses that the choice context is not limited to the choice set but includes also the alternatives that the decision maker expects to find in the current choice setting (Kahneman and Miller 1986). As we stressed previously, this aspect seems relevant to understanding several phenomena. For example, in the Thaler beer example, framing subjects with a specific context (resort or store) makes them think about the price they could expect in that context. In the Hastings and Shapiro example, the consumer approaches the gas station having in mind a price expectation for gas. The logic of our model implies that, once evoked, expected prices shape choice by affecting the salience ranking of different product attributes.

To see how these effects may work, we incorporate expectations in our definition of context. In a straightforward way, we assume that the choice context consists of the goods in the choice set together with those same goods at their rationally expected prices.

Definition 3. The choice context is the set $\mathbf{C}_{\text {cont }}=\mathbf{C} \cup \mathbf{C}_{e}$, where $\mathbf{C}$ is the externally given choice set and $\mathbf{C}_{e}=\left\{\left(q_{k}^{e}, p_{k}^{e}\right)\right\}_{k=1, \ldots, N}$ is the set of goods the consumer expects to find in the choice setting. We make the following assumptions:

i. For each $\left(q_{k}, p_{k}\right) \in \mathbf{C}$, there is a $\left(q_{k}^{e}, p_{k}^{e}\right)$ whose expected quality satisfies $q_{k}^{e}=q_{k}$ and whose expected price $p_{k}^{e}$ is the rational expectation of $p_{k}$, namely, $p_{k}^{e} \equiv \mathbb{E}\left[p_{k}\right]$.

ii. The choice context is summarized by a reference $\operatorname{good}(\bar{q}, \bar{p})$, where the reference (or normal) levels of quality and price are their average values in $\mathbf{C}_{\text {cont }}$, namely, $\bar{q}=(1 / N) \sum_{k} q_{k}$ and $\bar{p}=$ $(1 / 2 N) \sum_{k}\left(p_{k}+p_{k}^{e}\right)$.

This definition captures the idea that the choice situation brings to the consumer's mind "normal" prices, which then shape the consumer's reference price (Kahneman and Miller 1986). Assuming rational expectations is an intuitive and model-consistent way to capture normal prices, although it is not universally applicable. ${ }^{17}$

${ }^{17}$ In some settings, the prices rationally expected in a specific situation might be perceived as being far from normal, for instance, the price of a tuna sandwich at an airport or 
Definition 3 has two important implications. First, when actual prices coincide with price expectations (i.e., when $p_{k}=\mathbb{E}\left[p_{k}\right]$ for all $k$ ), the reference good in the choice context coincides with the average good in the choice set. As a result, the consumer behaves as if the choice context and the choice set coincide, $\mathbf{C}_{\mathrm{cont}}=\mathbf{C}$. In this sense, our previous analysis is directly applicable to deterministic settings or to lab experiments in which, unless explicitly primed, consumers have no experience to base their forecasts on.

Second, when expected and actual prices differ, the model uncovers an important new effect: the salience of price is determined not only by comparing prices across goods but also by comparing actual prices with expected prices. Even if the price of a good is similar to that of other goods, it may be salient if it is unexpectedly high. The salient thinker's attention is drawn not just to differences between available options but also to the surprising features of the environment. ${ }^{18}$

\section{A. Price Shifts: Diminishing Sensitivity versus Ordering}

We now provide a general characterization of the effects of price changes in our model. We show how the model generates two seemingly contradictory implications: lower price sensitivity at higher price levels but higher price sensitivity after unexpected price hikes. These results are due to the tension between the basic forces of diminishing sensitivity and ordering.

Take the choice context $\mathbf{C}_{\text {cont }}$ as given and consider the effect on salience of a marginal price increase in a proper subset of $\mathbf{C}_{\text {cont }}$. Here $\mathbf{C}_{\text {cont }}$ includes both available goods and the same goods at expected prices. Let us partition $\mathbf{C}_{\text {cont }}$ into two subsets, $\mathbf{C}_{\mathbf{F}}$ and $\mathbf{C}_{\mathbf{C}}$, such that $\mathbf{C}_{\mathbf{F}}$ is the set of goods for which price is held fixed and $\mathbf{C}_{\mathrm{C}}$ is the set of goods whose prices uniformly increase. Formally, suppose that the prices for goods $k$ in $\mathbf{C}_{\mathrm{C}}$ are increased from $p_{k}$ to $p_{k}+\Delta$, where $\Delta$ is a marginal uniform price increase for all goods in $\mathbf{C}_{\mathbf{C}}$. Depending on the "experiment," $\mathbf{G}_{\mathbf{C}}$ can include actual prices, expected prices, or both. The following cases are of particular interest:

of a hotdog at a baseball game. Assuming rational expectations strikes a balance between psychological precision and testability of the model.

${ }^{18}$ Our use of rational expectations is reminiscent of Koszegi and Rabin's (2006) model, in which reference points coincide with expectations, but has two important differences. First, in our model, expectations are fully exogenous in that they are determined by the entire choice context, and not by what is actually chosen. This simplifies the model and facilitates its application because it requires the modeler to know only the exogenous empirical distribution of prices. Second, our mechanism relies on salience relative to the reference good, and not on loss aversion. These two forces often act in directly opposite directions, for instance, when a good's quality is salient. 
a. Expected price hikes, as in the store versus restaurant example: In this case, both actual and expected prices change, so that $\mathbf{C}_{\mathbf{C}}=\mathbf{C}$ and $\mathbf{C}_{\mathbf{F}}=\varnothing$.

$b$. Unexpected price hikes: Here only actual prices change while expected prices stay constant in the choice context, so that $\mathbf{C}_{\mathrm{C}}=\mathbf{C}_{\text {choice }}$ and $\mathbf{C}_{\mathbf{F}}=\mathbf{C}_{e}$.

$c$. Relative price changes: Only a subset of actual (and perhaps expected) prices changes. Here $\mathbf{C}_{\mathrm{C}}$ is the subset of actual (and expected) goods whose relative price changes, while $\mathbf{C}_{\mathrm{F}}$ contains all remaining (actual and expected) goods.

Denote by $\eta$ the fraction of goods in the choice context that belong to $\mathbf{C}_{\mathrm{C}}$. Denote by $\bar{p}_{\mathrm{F}}$ the average price in subset $\mathbf{C}_{\mathrm{F}}$, by $\bar{p}_{\mathrm{C}}$ the average price in $\mathbf{C}_{\mathrm{C}}$, and by $\bar{p}$ the reference (average) price in $\mathbf{C}_{\text {cont }}$, where all prices are computed with $\Delta=0$. In example $a, \eta=1$ and $\bar{p}_{\mathrm{C}}=\bar{p}$. In example $b, \eta$ $=1 / 2$ and $\bar{p}_{\mathrm{F}}=\bar{p}_{\mathrm{C}}=\bar{p}$. In example $c$, average prices depend on the specific goods considered and $\eta$ may be small, close to zero. We then show the following proposition.

Proposition 5. Suppose that $\bar{p}_{\mathrm{C}} \geq \bar{p}$. Then a uniform marginal increase $\Delta$ in the prices of goods in $\mathbf{C}_{\mathrm{C}}$ boosts the salience of price for the most expensive good in $\mathbf{C}_{\mathrm{C}}$ only if

$$
\frac{p_{\mathrm{C}}^{\max }-\bar{p}_{\mathrm{C}}}{\bar{p}_{\mathrm{F}}}<\frac{1-\eta}{\eta},
$$

where $p_{\mathrm{C}}^{\max }$ is the highest price in $\mathbf{C}_{\mathrm{C}}$. If $\eta=1$, price salience falls for all goods in $\mathbf{C}_{\mathrm{C}}$.

Take a category of goods that are originally at least as expensive as average (i.e., $\bar{p}_{\mathrm{C}} \geq \bar{p}$ ). A uniform marginal increase in the prices in this category boosts the salience of price for its most expensive membersand thus increases the consumer's price sensitivity for these goodsprovided that the category affected by the price hike is sufficiently small, namely, $\eta$ is small.

The size $\eta$ of the category affected by the price hike modulates the strength of ordering versus diminishing sensitivity. When the category is large, the price hike induces a commensurate increase in the reference price $\bar{p}$. As a consequence, the price differential between category prices and the reference price does not grow significantly. Diminishing sensitivity dominates, equation (10) does not hold, and a price increase reduces the salience of price. This is what happens in the case of fully expected price hikes, such as in setting $a$ above: actual and expected prices uniformly increase $(\eta=1)$ and price salience falls for all goods, includ- 
ing the very expensive ones. The consumer's price sensitivity is reduced for all goods.

Suppose, in contrast, that the category affected by the price hikes is small (in the limit, $\eta \approx 0$ ). Now the reference price $\bar{p}$ is only modestly affected by the price hike. As a consequence, the price differential between category prices and $\bar{p}$ grows disproportionately. In this case, ordering dominates, equation (10) holds, and a price increase boosts the salience of price. The surprising price hike of setting $b$ above falls into this case: only actual prices increase while expected prices stay constant (so $\eta=1 / 2$ ). Because the prices of the available goods are very high relative to the reference price, the most expensive goods become price salient. This increases the consumer's price sensitivity to these goods.

As illustrated in setting $c$, this phenomenon describes consumers' reaction not only to surprises in overall price levels but also to relative price changes. Imagine, for instance, a consumer choosing among different qualities of Bordeaux wines. Equation (10) says that as the price of Bordeaux wines uniformly increases, the consumer is more likely to substitute toward cheaper Bordeaux (or potentially to leave the category altogether) if Bordeaux wines are, on average, expensive and display relatively low price dispersion.

In sum, proposition 5 provides the central comparative statics on how price sensitivity depends on context: consumers are relatively insensitive to price differences among goods at expected high prices, while they are very price sensitive when faced with unexpected price increases. Furthermore, our model yields testable predictions on whether price hikes boost or dampen the demand for quality, depending on the magnitude of the price hike and on the market structure (measured by $\eta$ and price dispersion $p_{\mathrm{C}}^{\max }-\bar{p}_{\mathrm{C}}$ in eq. $\left.[10]\right)$.

To illustrate the workings of proposition 5, consider again the wine example from the introduction. As the salient thinker opens the wine list at his favorite restaurant, he forms rational expectations of the prices he may find. Suppose that these expectations are given by $p_{h}^{e} \equiv \mathbb{E}\left[p_{h} \mid\right.$ rest $]$ $=60$ and $p_{l}^{e} \equiv \mathbb{E}\left[p_{l} \mid\right.$ rest $]=50$. To account for the possibility that actual prices are higher than expected, write actual prices as $p_{h}^{\text {rest }}=60+s$ and $p_{l}^{\text {rest }}=50+s$, where $s \geq 0$ is the price surprise. If $s=0$, we are in case $a$, where $\eta=1$ and price expectations coincide with actual prices. If instead $s>0$, we are in case $b$, where $\eta=1 / 2$ and the consumer faces an unexpected price increase. The consumer's choice context depends on the price shock $s$ :

$$
\mathbf{C}_{\text {rest }}= \begin{cases}h^{\text {rest }}= & (30,60+s) \\ l^{\text {rest }}= & (20,50+s) \\ h^{e, \text { rest }}= & (30,60) \\ l^{\text {,rest }}= & (20,50) .\end{cases}
$$


The reference wine is $(\bar{q}, \bar{p})=(25,55+(s / 2))$. The high-end wine $h^{\text {rest }}$ still yields above-average quality, but it may now exhibit a lower than average quality/price ratio. This is indeed the case provided that

$$
\frac{30}{60+s}<\frac{25}{55+(s / 2)} \Leftrightarrow s>15 .
$$

As implied by proposition 5 , if the price surprise is sufficiently large, the high-end wine becomes price salient. This greatly reduces the value of $h^{\text {rest }}$ as perceived by the salient thinker. This price surprise might also render the low-end wine price salient, but it unambiguously reduces the relative valuation of the high-end wine, inducing the consumer to choose the lowend wine. When the consumer finds wines at the restaurant to be unexpectedly pricey, he switches to lower-quality wine, excessively reducing his demand for quality relative to the rational case.

Compare this result with the case in which restaurant prices are high but fully expected, namely, $s=0$. In that case, the consumer is adapted to higher wine prices, and by diminishing sensitivity, he focuses on quality and chooses the high-quality wine. The key difference with the previous case is that now high restaurant prices are fully expected and so do not draw the consumer's attention.

To conclude the analysis, consider what happens when restaurant prices are surprisingly low, namely, $s<0$. In this case, equation (12) is harder to satisfy. Relative to historical prices, the high-end wine now has a high quality/price ratio. Thus, unexpectedly low prices tend to draw the consumer's attention to quality, reducing his price sensitivity. The high-quality wine is now chosen over its low-quality competitor. Interestingly, this effect holds only for moderate price drops. If the price drop is sufficiently drastic, the consumer's attention is necessarily drawn to prices, which again favors the low-quality wine. Our model thus exhibits an asymmetry whereby unexpected price hikes always induce consumers to substitute toward cheaper goods while unexpected price declines tend to, but do not always, induce consumers to substitute toward more expensive goods.

This intuition helps account for the evidence in Hastings and Shapiro (2013). They show that consumers react to parallel increases in gas prices by switching to cheaper (and lower-quality) gasoline. In online Appendix D, we show how the broad patterns in the demand for gasoline documented by Hastings and Shapiro emerge from our model, namely, that the share of regular gasoline rises with gas prices, as consumers' price sensitivity is asymmetrically boosted by price hikes. The intuition follows from proposition 5: assuming that gas prices follow a random walk, the rationally expected prices coincide with the last observed prices. If the consumer observes surprisingly high prices for all gas grades, the price of 
the high-quality grade becomes even more salient, making the consumer relatively more sensitive to price differences and more likely to switch to lower-octane, cheaper gas. If instead the consumer observes surprisingly low prices for all gas grades, then, as long as the price drop is not too steep, the price of the high-quality grade approaches the reference price and is therefore less likely to be salient, making the consumer less sensitive to price differences and more likely to switch to higher-octane, more expensive gas. ${ }^{19}$

\section{B. Manipulation of Expectations}

The willingness to pay (WTP) for quality $q$ is defined as the maximum price at which the consumer is willing to buy $q$ instead of sticking to the outside option of no consumption $\left(q_{0}, p_{0}\right)$. Typically $q_{0}=p_{0}=0$. In standard theory, knowledge of $q$ and of $q_{0}$ and $p_{0}$ are sufficient to determine WTP for $q$ (assuming quasi-linear utility, as we do here). In contrast to this prediction, evidence suggests that the WTP for a good can be influenced by contextual factors (Thaler 1985). Our model indicates that WTP may vary across contexts that differ in price expectations.

Formally, suppose that the consumer must state his WTP for quality $q$ while expecting a good $\left(q, p^{e}\right)$, where $p^{e}$ is the expected price at which quality $q$ is sold. The consumer evaluates the $\operatorname{good}(q, p)$ at a price $p$, so his choice context is $\mathbf{C}_{\text {cont }} \equiv\left\{(0,0),\left(q, p^{e}\right),(q, p)\right\}$, where $(0,0)$ is the outside option of not consuming $q$. We define the consumer's WTP for $q$ in this context as

$$
\begin{gathered}
\operatorname{WTP}\left(q \mid\left(q, p^{e}\right)\right)=\sup p \\
\text { subject to } u^{S}\left((q, p) \mid \mathbf{C}_{\text {cont }}\right) \geq u^{S}\left((0,0) \mid \mathbf{C}_{\text {cont }}\right) .
\end{gathered}
$$

WTP is still defined as the maximum price $p$ that the consumer is willing to pay for $q$ as opposed to getting the outside option $(0,0)$, but the superscript $S$ indicates that the consumer's preferences are distorted by salience. Crucially, different values of the good's price $p$ can alter the salience of its attributes, changing the consumer's valuation. Thus, maxi-

${ }^{19}$ Hastings and Shapiro calibrate a salience model and evaluate its predictions. An important aspect of the calibration is the measurement of quality, which shapes the salience of quality to the consumer, affecting his reaction to given price changes. This particular setting suggests two measures of quality, one numerical ( 87 vs. 91 octane rating) and one descriptive (regular vs. premium gasoline). The descriptive measure likely conveys a more salient quality difference than a 4 percent difference in octane rating. Lacking guidance on which measure to use, in many applications it may be useful to treat quality as a latent variable (even when certain objective proxies for quality are available) to be estimated with the remaining parameters of the model. 
mization in (13) tends to select a price $p$ such that good $(q, p)$ 's quality is salient.

In the choice context $\mathbf{C}_{\text {cont }}$, the reference good has quality $\bar{q}=q \cdot(2 / 3)$ and price $\bar{p}=\left(p+p^{e}\right) / 3$. We can then show the following proposition.

Proposition 6 . The consumer's WTP for $q$ depends on the expected price $p^{e}$ as follows:

$$
\mathrm{WTP}\left(q \mid\left(q, p^{e}\right)\right)= \begin{cases}\delta q & \text { if } p^{e} \leq \delta q \\ p^{e} & \text { if } \delta q<p^{e} \leq \frac{1}{\delta} \cdot q \\ \frac{q}{\delta} & \text { if } \frac{1}{\delta} \cdot q<p^{e} \leq \frac{7}{2 \delta} \cdot q \\ \delta q & \text { if } p^{e}>\frac{7}{2 \delta} \cdot q .\end{cases}
$$

As $\delta \rightarrow 1$, the WTP tends to $q$ and becomes independent of context $p^{e}$.

The price expectation $p^{e}$ affects WTP only if the consumer is a salient thinker, that is, if $\delta<1$. Importantly, for $p^{e} \leq(7 / 2 \delta) q$, the consumer's WTP weakly increases in the expected price $p^{e}$. In contexts in which quality is more expensive, the consumer is willing to pay a higher price $p$ and still view quality as salient. ${ }^{20}$ Through salience, a higher price $p^{e}$ acts like an anchor, increasing WTP. This mechanism can explain Thaler's beer experiment, in which subjects stated a higher WTP when the place from which a beer is bought was specified to be a nearby resort hotel than when it was a nearby grocery store. This follows from proposition 6 because the expected price at the resort is higher than that in the store (i.e., $p_{\text {resort }}^{e}>p_{\text {store }}^{e}$ ). Intuitively, when thinking of the high expected price at the resort, the salient thinker is willing to pay a high price for the beer and still perceive quality as salient. When thinking of the low expected price at the store, however, the salient thinker is not willing to pay a high price for the beer because such a high price would be salient at the store.

Interestingly, proposition 6 suggests that when the reference price is extremely high, this effect vanishes. In this respect our model yields a different prediction than the one based on transaction utility. If the expected price is too high, formally if $p^{e} \gg q / \delta$, price becomes salient and the consumer's WTP drops. Intuitively, if the consumer expects beer at resorts to be outrageously expensive, then he focuses on the high prices at the resort, which causes his valuation for beer to drop in this context. As a result, the consumer refuses to buy a beer even if the price is close to his

${ }^{20}$ Put differently, as $p^{e}$ increases, the consumer perceives $(q, p)$ as a good deal even at higher prices $p$. 
true valuation. In fact, this causes the consumer's WTP to drop below his true valuation. The WTP in (14) is graphically represented in figure 1.

\section{Falsifiable Predictions of the Model}

In the previous sections we characterized the predictions of the model that hold when the choice context $\mathbf{C} \cup \mathbf{C}_{e}$ is known. In realistic settings, though, only some features of the choice context (e.g., the prices of goods in the choice set) are readily available to an observer. Other inputs to the model such as the precise quality levels or the expected prices are difficult to observe. We now highlight some falsifiable predictions of our model when the researcher does not have direct knowledge of quality levels and/or expected prices. We study predictions that hold under any salience function satisfying assumption 1 and consider two scenarios:

1. The choice context coincides with the choice set. Quality rankings are observed but quality levels are not.

2. The choice context includes expected prices. Quality rankings are observed but neither quality levels nor expected prices are observed.

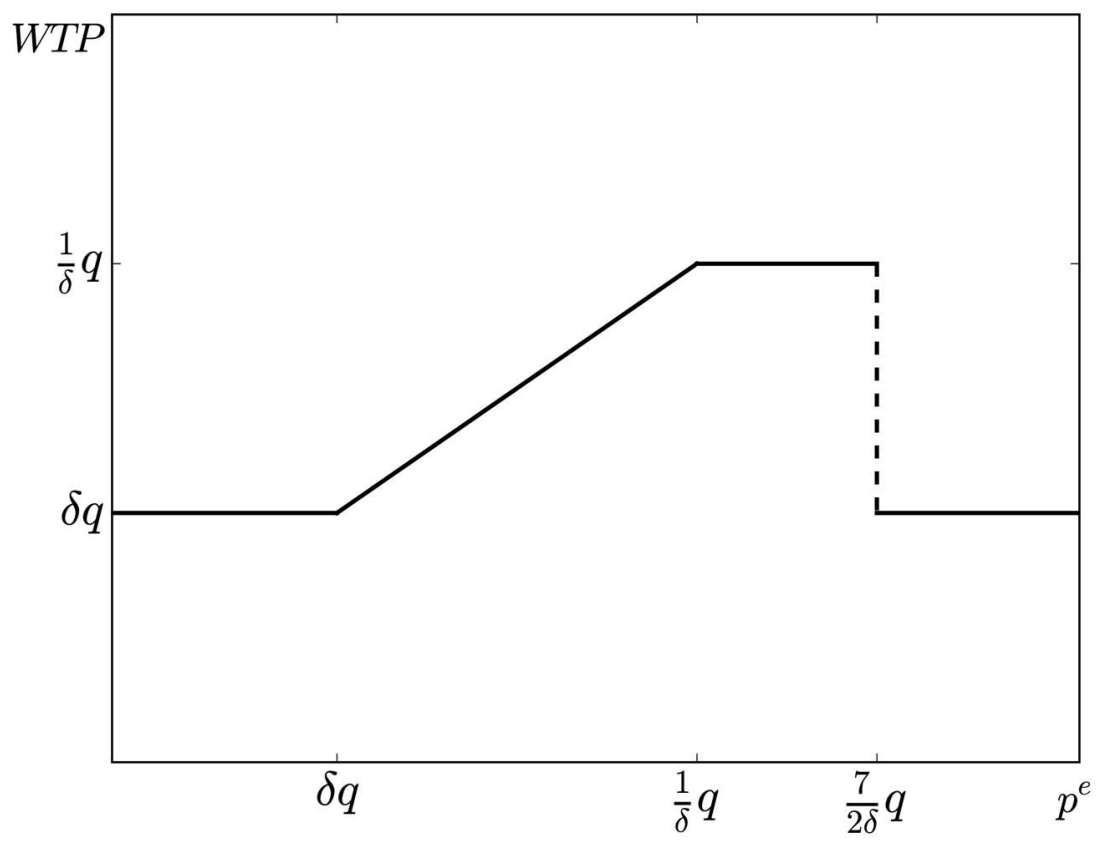

Fig. 1.-Willingness to pay for $q$ as a function of expected price $p^{e}$ 
Scenario 1 holds in lab experiments, in which subjects do not have strong preexisting expectations about prices (unless these are induced by the experimenter), but it also holds in the field when consumers are familiar with the available goods and prices can (roughly) be assumed to be stable. One interesting property of this scenario is that even if the quality $q$ of a good is not directly observable, it can be recovered by the researcher by running a pricing experiment eliciting a subject's WTP. In such an experiment, the choice set is given by $\mathbf{C} \equiv\{(q, p),(0,0)\}$, where $p$ is the asking price. Because the choice set coincides with the choice context, the reference good is $(\bar{q}, \bar{p}) \equiv(q / 2, p / 2)$. Under a homogeneous of degree zero salience function, we have that

$$
\sigma(q, q / 2)=\sigma(p, p / 2)=\sigma(1,1 / 2) .
$$

That is, quality and price are equally salient for any asking price $p$. We now make the following remark.

Remark 1. In the choice context $\mathbf{C} \equiv\{(q, p),(0,0)\}$, valuation is not distorted by salience and the salient thinker's willingness to pay for $q$ is $\mathrm{WTP}=q$.

When a good is evaluated in isolation and without price expectations, the WTP for it equals its quality level, just as in the rational model. The absence of salience distortions is ensured by the assumption that the outside option is given by $(0,0)$ (together with homogeneity of the salience function). This assumption is realistic in the sense that salience is defined over product attributes, and the option of not buying the good is naturally framed as zero price and quality. WTP is best elicited in a controlled laboratory setting, where this framing can be made explicit.

The ability to recover quality through WTP is important because it can in principle allow researchers to directly test the predictions of the model, such as those highlighted in proposition 4 , in deterministic or lab settings in which price expectations do not play a role.

But even if WTP cannot be readily elicited through price experiments (e.g., in certain field applications), information on quality ordering still yields falsifiable predictions based on price changes (which are observable) in situations in which the choice context coincides with the choice set. In particular, consider scenario 1 , in which the choice context coincides with the choice set and the researcher observes only prices and the ranking of qualities. The simplest setting consists of a binary choice between a high-quality and a low-quality good. In this case, one can directly test the prediction of corollary 1 , whereby the demand for the high-quality good should (weakly) rise after an expected uniform price increase. In fact, this prediction depends only on knowledge of (i) prices and (ii) the quality ranking of the two goods. 
With more than two goods the analysis is more complicated, but our model still yields testable predictions. Suppose that the choice set has $N>2$ goods and that the researcher observes quality rankings $q_{1}<q_{2}$ $<\cdots<q_{N}$ and prices $p_{1}+\Delta<p_{2}+\Delta<\cdots<p_{N}+\Delta$, where $\Delta \geq 0$. Because we are in scenario 1 , the researcher observes the reference price $\bar{p}=$ $(1 / N) \sum_{k} p_{k}+\Delta$ and can manipulate all prices that determine $\bar{p}$. We can then prove the following prediction.

PREDiction 1. Let $p_{j}>(1 / N) \sum_{k} p_{k}>p_{i}$, and suppose that a consumer chooses good $i$ when $\Delta=0$ and good $j>i$ when $\Delta=\Delta^{*}>0$. Then, keeping $\Delta=0$, if the prices of all goods $k \neq i, j$ experience a uniform increase so that the new average price is equal to $p_{j}$, then good $j$ is preferred to good $i$, so the latter cannot be chosen.

This result highlights the mechanics underlying violations of the IIA in our model. It exploits the fact that the consumers' price sensitivity toward the high-quality good $j$ is reduced under two distinct patterns of price increases: if a uniform price hike reduces the consumer's price sensitivity and induces him to switch to higher quality $q_{j}>q_{i}$, then the consumer must choose $q_{j}$ as its price becomes less salient. As a result, IIA can be violated by changing the prices of "irrelevant" goods $k \neq i, j$ so as to render the quality $q_{j}$ salient. The best way to implement this procedure experimentally is to rely on between-subject tests in order to avoid a situation in which price manipulations influence the formation of price expectations in the lab. The prediction can also be tested in a withinsubject paradigm, but only when it is possible to ensure that subjects fully expect the actual price shifts (as in the store vs. restaurant example).

We now turn to the case in which price expectations are present. While in many cases reasonable proxies for price expectations can be found, we focus on the most conservative case in which the researcher cannot observe them. This is the case of scenario 2. Now the location of the reference price is not known, which makes it difficult to control the salience ranking of specific goods. This makes testing our model more difficult. However, proposition 6 provides a clue on how the demand for quality $q$ depends on its price $p$, given an arbitrary expected price $p^{e}$ : the demand for quality is inverse-U-shaped in the sense that a good's valuation is maximized when current prices are close to expected prices (and quality is salient) and is lower otherwise (when price is salient). ${ }^{21}$ In the simple case of pairwise choice, this behavior leads to the testable prediction 2.

${ }^{21}$ In particular, an increase in the observed price levels increases the salience of price provided that observed prices are at or above the expected prices. This is the case in the Hastings and Shapiro example of demand for gas, where expected prices are known (they are specified to be the past-period prices) and price shifts are defined relative to expectations. However, if observed prices are low relative to expectations, then a price hike boosts the relative salience of quality by bringing observed prices closer to expected prices. Thus, if expectations are known (as in scenario 1) but do not necessarily coincide with observed prices (as in scenario 2), the model has further testable predictions. 
Prediction 2. Suppose that the choice set consists of two goods $(N=2)$, with $q_{1}<q_{2}$ and $p_{1}<p_{2}$, and that the expected prices are fixed but not observed. Under uniform price shifts, $p_{k} \rightarrow p_{k}+\Delta p$ for $k=1,2$ and $\Delta p>0$, there can be at most two changes in pairwise choice. If there exist price shifts $\Delta p_{a}<\Delta p_{b}<\Delta p_{c}$ such that good 1 is chosen at prices $p_{k}$ $+\Delta p_{a}$ and $p_{k}+\Delta p_{c}$ but good 2 is chosen at prices $p_{k}+\Delta p_{b}$, then good 1 is chosen at any price level $p_{k}+\Delta p$ satisfying $\Delta p \in\left[0, \Delta p_{a}\right]$ or $\Delta p \geq \Delta p_{c}$.

As a function of the price level, the quality of the chosen good is either flat (if the same good is chosen under all salience rankings), monotonic, or inverse-U-shaped (the case considered in the prediction). Prediction 2 can be interpreted as describing preference reversals between two goods within arbitrary choice sets. In this sense, data on consumers' full preference rankings, rather than simply choice, provide a better test of the model when expectations are not observed.

\section{Misleading Sales}

To illustrate how our model can clarify field evidence on context effects, we develop an application to sales. Retailers frequently resort to sales events as a means to sell their products. In 1988, sales accounted for over 60 percent of department store volume (Ortmeyer et al. 1991). The standard explanation for sales is price discrimination: sporadic sales allow retailers to lure low-willingness-to-pay customers, whereas high-willingnessto-pay customers who cannot wait for a sale buy at the higher regular prices (Varian 1980; Sobel 1984; Lazear 1986). It is probably true that lowwillingness-to-pay customers tend to sort into sales events, but the high frequency and predictability of sales cast some doubt on the universal validity of the price discrimination hypothesis. In particular, there is some concern that retailers may deliberately inflate regular prices in order to lure consumers into artificial sales events. The Pennsylvania Bureau of Consumer Protection has successfully pursued retailers for advertising misleading sales prices. In Massachusetts, regulatory changes have tightened rules for price comparison claims, for example, requiring that retail catalogues state that the "original" price is a reference price and not necessarily the previous selling price.

In this section we show that salience-and in particular the logic of decoy effects - can shed light on these "misleading sales" events. We highlight two new predictions of our salience-based model of sales:

- In a store selling different qualities, misleading sales boost demand only for high-quality goods, and this occurs at the expense of demand for lower-quality goods.

- Misleading sales boost demand only for nonstandard goods. 
Specifically, suppose that a consumer is considering whether or not to buy a good of quality $q$ and price $p$ in a store. The good is nonstandard in the sense that it has no substitutes and is available only at this store; we later consider the case of standard goods, which can be easily found at different stores. The choice set faced by the consumer is $\mathbf{C}_{0} \equiv$ $\{(0,0),(q, p)\}$, where $(0,0)$ is the outside option of not buying the good. In this case, the consumer's valuation of the good is rational, and the maximum price he can be charged for the good is his true valuation, namely, $p=q$.

Suppose now that there is a sale event in the store. By a sale event we mean that, with some probability $\pi$, the consumer is offered a given quality $q$ at the sale price $p_{s}$ rather than at the full regular price $p_{f}>p_{s}$. Since the consumer has rational expectations regarding the possibility of sales, his expected price is $\mathbb{E}[p]=\pi p_{s}+(1-\pi) p_{f}$. When deciding whether or not to buy the good, this expected price becomes part of the consumer's choice context, $\mathbf{C}_{\text {sale }} \equiv\left\{(0,0),\left(q, p_{s}\right),(q, \mathbb{E}[p])\right\}$.

Consider the standing of the option $\left(q, p_{s}\right)$ in the new choice context $\mathbf{C}_{\text {sale }}$. The salience of quality is $\sigma(q, 2 q / 3)$, while the salience of price is $\sigma\left(p_{s},\left(\mathbb{E}[p]+p_{s}\right) / 3\right)$. The central implication is that the retailer can manipulate the salience of price by manipulating the price discount $p_{s} / p_{f}$. In particular, we can establish the following proposition.

Proposition 7. The retailer can charge a sale price equal to the consumer's maximum willingness to pay, $p_{s}=q / \delta$, by setting the full price in the interval $p_{f} \in(q / \delta, q / \delta \cdot(7-2 \pi) /(2-2 \pi))$.

By artificially inflating the regular price of the good and by offering at the same time a generous discount, the retailer can extract up to the maximum consumer valuation $q / \delta$. The reason is that the consumer views the discount as a good deal, boosting his valuation of quality. This misleading sales logic suggests that firms generate most returns during the sales events themselves, consistent with Ortmeyer et al. (1991). This is in contrast with psychological models of sales based on loss aversion (e.g., Heidhues and Koszegi 2008), where firms make money on sales at regular prices, and sales events are used as a potentially unprofitable bait to generate loss aversion in consumers.

We now examine the robustness of this sales mechanism to the specification of the choice set. In particular, we consider the case in which the outside option is a different good, with positive quality and price. This leads us to our first prediction, namely, that a "misleading sale" is effective only for a high-quality good.

Suppose that the store has a high-quality good $h=\left(q_{h}, p_{h}\right)$ and a lowerquality good $l=\left(q_{l}, p_{l}\right)$, where $q_{h}>q_{l}$ and $p_{h}>p_{l}$. For the sake of illustration, we assume that the prices $p_{l}$ and $p_{h}$ at which these goods are sold are exogenously fixed (e.g., by the producer). The store, however, can try 
to influence which good is sold by adopting a misleading sales policy. In the case of the high-quality good, this amounts to making the good occasionally available (say, with probability $1-\pi$ ) also at a full price $p_{f h}>p_{h}$. Similarly, for the low-quality good, the store can set a full price $p_{f l} \in\left(p_{l}, p_{h}\right)$ with the same probability.

Proposition 8. Let $q_{h}-\delta p_{h}>q_{l}-\delta p_{l}$ and $\delta q_{h}-p_{h}<\delta q_{l}-p_{l}$, so that when goods are at "sale" prices $p_{l}$ and $p_{h}$, good $h$ is sold if and only if it is quality salient. Then

i. regardless of the consumer's choice in the absence of a sale, the retailer can sell good $h$ by holding a sale on it where the full price $p_{f h}$ is suitably chosen;

ii. if the consumer chooses $h$ in the absence of a sale, there exists no full price $p_{f l} \in\left(p_{l}, p_{h}\right)$ for $l$ that makes $h$ price salient, and $l$ be chosen, in the context of the sale.

It is always possible to engineer sales inducing the salient thinker to overvalue the high-quality good $h$ relative to $l$, but not the reverse. The same mechanism underlying the asymmetry of the decoy effect is at work here since the high regular price effectively acts as a decoy. Holding a sale on the good with the lowest quality/price ratio unambiguously decreases the quality/price ratio of the reference good. This effect reinforces the salience of quality for the high-quality good and the salience of price for the low-quality good (since price is its relative advantage), increasing the relative overvaluation of the former.

Our novel prediction on the asymmetry in the effectiveness of salesas well as the implication that demand for the high-quality good grows at the expense of that for the low-quality competitor-has also been documented in the field. In their review on the literature on promotions, Blattberg, Briesch, and Fox (1995) present this asymmetry as one of the stylized facts in the field of marketing.

Consider now our second prediction, namely, that sales are unlikely to work with standard goods, for which market prices are well known. A consumer wishes to purchase a standard good of quality $q$, for instance, a metro ticket. There are $N>1$ potential sellers of the good. Suppose that each of these sellers implements the same misleading sales policy consisting of a regular price $p_{f}$ and a sale price $p_{s}$, each occurring with probability $\pi=1 / 2$, and where $p_{f} / p_{s}=k \in(1,6)$ (see proposition 7 above).

In this case, the consumer's choice context consists of $2 N$ goods (two goods for each of the $N$ sales) and the outside option of not buying $(0,0)$. Formally, $\mathbf{C}_{\text {sale }}$ is given by $\left\{(0,0),\left(q, p_{s}\right), \ldots,(q, \mathbb{E}[p])\right\}$, where $\left(q, p_{s}\right)$ and $(q,-\mathbb{E}[p])$ are repeated $N$ times, and $\mathbb{E}[p]=p_{s} \cdot(1+k) / 2$. For the items on sale, then, the salience of quality is $\sigma(q, q[2 N /(2 N+1)])$, and that of 
price is $\sigma\left(p_{s}, p_{s}[N \cdot(3+k) / 2] /(2 N+1)\right)$. Owing to homogeneity of degree zero, these expressions imply that when the number of sellers is sufficiently large, namely, when

$$
N>\frac{2+\sqrt{3+k}}{k-1},
$$

the items on sale have salient price, that is,

$$
\sigma\left(q, q \frac{2 N}{2 N+1}\right)<\sigma\left(p_{s}, p_{s} \frac{N \cdot(3+k) / 2}{2 N+1}\right),
$$

rather than salient quality as in the nonstandard good case of proposition 7 .

This result is intuitive and holds for any magnitude $k$ and frequency $\pi$ of the sale. As the number of sellers $N$ increases, the average quality $\bar{q}=q[2 N /(2 N+1)]$ in the choice set gets arbitrarily close to the quality $q$ of the standard good. As a result, quality becomes nonsalient. By contrast, the price variability generated by sales renders prices salient, increasing the consumer's price sensitivity above its rational counterpart. As a result, when deciding where to buy a standard good, the salient thinker focuses on price because price is the attribute that varies most across sellers (almost by definition of standard goods)! This implies that a generalized policy of misleading sales does not work in the case of standard goods: because the inherent price variation induces consumers to focus on prices, the sales event reduces their willingness to pay, so that the overall demand for the standard good falls.

\section{Conclusion}

We combine two ideas to explain a wide range of experimental and field evidence regarding individual choice, as well as to make new predictions.

The first idea is that choices are made in context and that, in particular, goods are evaluated by comparison with other goods the decision maker is thinking about. This idea is intimately related to Kahneman and Tversky's (1979) concept of reference points and is also central to related studies of choice by Tversky and Kahneman (1991), Tversky and Simonson (1993), Bodner and Prelec (1994), and Koszegi and Rabin (2006). In our model, context is often determined by the choice set itself, and the reference good relative to which the options are evaluated has the average characteristics of all the goods in the choice set. In some examples, expectations about prices also influence what decision makers are thinking about, and the choice context shaping the reference good is larger 
than the choice set. To discipline the model, we assume that price expectations are rational, but this assumption may need to be revised in some applications.

The second idea, which extends our earlier work on choice under risk (Bordalo et al. 2012), holds that the salience of each good's attributes relative to the reference good, such as its quality and price, determines the attention the decision maker pays to these attributes as well as their weight in his decision. We argue that ordering and diminishing sensitivity are the two critical properties of salience that together help account for a broad range of evidence.

We show that our model provides new insight into several puzzles of consumer choice. The model makes stark predictions for choice in experimental settings, in which the choice context is fully controlled by the experimenter. By showing how irrelevant alternatives change the reference good - and thus the salient attributes of existing alternatives - the model accounts for two well-known violations of independence of irrelevant alternatives, namely, decoy and compromise effects. But our mechanism also makes a novel prediction that decoy and compromise effects are asymmetric in that they differentially benefit more extreme goods (e.g., expensive, high-quality goods), a prediction that has strong experimental support. In the design of desirable goods, the model predicts a preference for some specialization as long as a minimum balance across attributes is provided.

By allowing expected prices to shape the reference good, the model has stark implications for responses to price changes. Consistent with previous research, salient thinkers may exhibit lower price sensitivity when price levels are high, but only when the high price level is fully expected. In contrast, the model makes the novel prediction that surprising price increases increase price sensitivity. In particular, the model accounts for context-dependent willingness to pay, exemplified by Thaler's celebrated beer example. Taken together, these predictions suggest that the salience mechanism can be seen as a simpler alternative to loss aversion in generating context effects.

We show how these results provide a unified way of thinking about field evidence previously described as mental accounting, in particular, describe how consumers react to changes in the prices of individual goods or whole categories of goods. We provide a novel explanation of Hastings and Shapiro's empirical finding that consumers substitute toward lowerquality gasoline when all gas prices rise while at the same time accounting for instances in which consumers substitute toward higher-quality goods when prices rise (e.g., the wine example). We also present a new theory of sales based on the idea that the original prices of goods put on sale serve as decoys that attract consumers to these goods. Our approach, unlike the standard model of sales, explains why firms often try to put goods 
on sale immediately after offering them first, so that "original" prices are in effect reference prices and not the previous selling price (leading to conflict with regulators). It also generates new predictions, such as that a store selling different qualities would put only high-quality goods on sale and that sales are most effective in boosting demand for nonstandard goods. We have noted throughout the paper a number of possible extensions and empirical tests, which we leave to future work.

\section{Appendix A}

\section{Proofs}

\section{Proof of Proposition 1}

The salience of $k$ 's quality is $\sigma\left(q_{k}, \bar{q}\right)$, while the salience of price is $\sigma\left(p_{k}, \bar{p}\right)$. Suppose that statement 1 holds so that $\sigma\left(q_{k}, \bar{q}\right)>\sigma\left(p_{k}, \bar{p}\right)$ if and only if $q_{k} / p_{k}>\bar{q} / \bar{p}$, namely, $q_{k} / \bar{q}>p_{k} / \bar{p}$. Consider the implications for $\sigma\left(q_{k}, \bar{q}\right)$. For any given values of $p_{k}, \bar{p}$, the condition $\sigma\left(q_{k}, \bar{q}\right)=\sigma\left(p_{k}, \bar{p}\right)$ is invariant under scaling of $q_{k}$ and $\bar{q}$, as it depends only on the ratio $q_{k} / \bar{q}$. As a result, $\sigma\left(q_{k}, \bar{q}\right)$ must depend only on this ratio and must be proportional to $\sigma\left(q_{k} / \bar{q}, 1\right)$. Setting $q_{k}=\bar{q}$ shows that the proportionality constant is one.

Suppose now that statement 2 holds. Then $\sigma\left(q_{k}, \bar{q}\right)=\sigma\left(q_{k} / \bar{q}, 1\right)$ and $\sigma\left(p_{k}, \bar{p}\right)=$ $\sigma\left(p_{k} / \bar{p}, 1\right)$, where both $q_{k} / \bar{q}$ and $p_{k} / \bar{p}$ are larger than one. By the ordering property of salience, then, quality is salient if and only if $q_{k} / \bar{q}>p_{k} / \bar{p}$. QED

\section{Proof of Proposition 2}

Consider a rational indifference curve characterized by $u(q, p)=q-p=u$. As in the text, order the elements of the choice set by increasing quality and price, so that $\left(q_{1}, p_{1}\right)$ is the cheapest good. The goods' quality/price ratios satisfy $q_{i} / p_{i}$ $=1+\left(u / p_{i}\right)$, and in particular, the reference $\operatorname{good}(\bar{q}, \bar{p})$ satisfies $\bar{q} / \bar{p}=1+(u / \bar{p})$. As in the text, we assume that $(\bar{q}, \bar{p})$ is not in the choice set.

1. $q_{1} / p_{1}>1$ when $u>0$, in which case the quality/price ratio is decreasing as price increases, and price is salient for all goods. The reason is that price is the relative advantage of cheap goods (whose prices are under $\bar{p}$ and have high quality/price ratios), while it is the relative disadvantage of expensive goods (whose prices are under $\bar{p}$ and have low quality/price ratios). Since the cheapest good is the best option along the salient price dimension, it is chosen and $k^{*}=1$. Formally, all goods are undervalued, $u^{S}\left(q_{i}, p_{i}\right)=2\left(\delta q_{i}-p_{i}\right) /(\delta+1)$, but the cheapest good is the least undervalued.

2. $q_{1} / p_{1}<1$ when $u<0$, in which case the quality/price ratio is increasing as price increases, and quality is salient for all goods. Since the most expensive good is the best option along the salient quality dimension, it is chosen and $k^{*}$ $=N$. Formally, all goods are overvalued, $u^{S}\left(q_{i}, p_{i}\right)=2\left(q_{i}-\delta p_{i}\right) /(1+\delta)$, but the highest-quality good is the most overvalued.

3. $q_{1} / p_{1}=1$ when $u=0$, in which case the quality/price ratio is constant along the indifference curve. Quality and price are equally salient for all goods. The 
salient thinker evaluates each good correctly (as the rational agent) and is indifferent between them. QED

Proof of Proposition 3

Consider a set of goods $\left(q_{k}, p_{k}\right)_{k=1 \ldots \ldots N}$ that lie on a rational indifference curve, $q_{k}-p_{k}=u$. Let $(\bar{q}, \bar{p})$ be the average quality and price in this set. The full choice set is $\left(q_{k}, p_{k}\right)_{k=0, \ldots N}$, which includes the outside option $\left(q_{0}, p_{0}\right)=(0,0)$. The reference good has quality $\bar{q} \cdot N /(N+1)$ and price $\bar{p} \cdot N /(N+1)$. While the quality/price ratio of the reference good is the same as in the absence of the outside option, namely, $\bar{q} / \bar{p}$, the reduction in the reference levels of quality and price may shift the location of the reference relative to some goods (of intermediate quality and price). This would affect the valuation of such goods and possibly the salient thinker's choice. We distinguish three cases.

1. $q_{1} / p_{1}<1$ when $u<0$, in which case the quality/price ratio is increasing as price increases, for $k \geq 1$ (recall that quality and price are increasing in the index $k$ ). Then the highest-quality $\operatorname{good}\left(q_{N}, p_{N}\right)$ is quality salient since it has aboveaverage quality and an above-average quality/price ratio. This implies that it is overvalued relative to, and thus preferred to, all other goods $k<N$ (regardless of the salience rankings of the latter). The condition $q_{N} / p_{N}>\delta$ ensures that good $\left(q_{N}, p_{N}\right)$ is also preferred to the outside option $(0,0)$.

2. $q_{1} / p_{1}>1$ when $u>0$, in which case the quality/price ratio is decreasing as price increases, for $k \geq 1$. Before exploring the salience rankings of the goods in the choice set, we outline the implications for choice. Suppose first that all goods have salient prices. Then good $\left(q_{1}, p_{1}\right)$ is overvalued relative to the other available goods $k \geq 2$, and the condition $q_{1} / p_{1}>1 / \delta$ ensures that it is preferred over the outside option as well. Now suppose that there are some goods whose quality is salient, and let good $\left(q_{k}, p_{k}\right)$ be the highest-quality good among them. Then $\left(q_{k}, p_{k}\right)$ is overvalued relative to all other options, including the outside option, and is chosen (recall that $q_{k}-p_{k}=u>0$ ). Intuitively, because the reference good lies "below" the rational indifference curve, $(\bar{q}-\bar{p}) \cdot N /(N+1)<u$, goods that are quality salient have a price similar to that of the reference good but a higher quality. Formally, such goods satisfy $q_{k} / p_{k}>\bar{q} / \bar{p}$ and $q_{k} \cdot p_{k}>\bar{q} \cdot \bar{p} \cdot N^{2} /(N+1)^{2}$ and, in particular, have a higher quality/price ratio than the reference good.

3. $q_{1} / p_{1}=1$ when $u=0$, in which case the quality/price ratio is the same for all $k \geq 1$. In particular, the reference good lies on the rational indifference curve, so that all goods have the same quality/price ratio and-as in the absence of the outside option-quality and price are equally salient for all goods. Because valuation is not distorted, the consumer is indifferent between the goods in the choice set. QED

Proof of Proposition 4

A sufficient condition for reversal between $l$ and $h$ is that good $h$ is chosen if and only if its relative advantage, namely quality, is salient. This means that $q_{h}-\delta p_{h}$ $>q_{l}-\delta p_{l}$ and also $\delta q_{l}-p_{l}>\delta q_{h}-p_{h}$. The first expression yields $\Delta u>-(1-$ 
$\delta)\left(p_{h}-p_{l}\right)$ and the second yields $\Delta u<(1-\delta)\left(q_{h}+q_{l}\right)$, where $\Delta u=\left(q_{h}-q_{l}\right)-$ $\left(p_{h}-p_{l}\right)$.

Consider case i. Since $q_{l} / p_{l}>q_{h} / p_{h}$, so that good $h$ has a relatively low quality/ price ratio, price is salient in $\{l, h\}$ and $l$ is chosen. If adding the decoy $d$ to the choice set makes $h$ quality salient, then the latter is preferred to $l$ in $\{l, h, d\}$. Good $h$ becomes quality salient in several different regimes: $(a)$ if $h$ has high quality and a high quality/price ratio relative to the reference good, $q_{h} / p_{h}>\bar{q} / \bar{p}, q_{h}>\bar{q}$ and $p_{h}>\bar{p} ;(b)$ if $h$ dominates the reference good, with higher quality and lower price, $q_{h} \cdot p_{h}>\bar{q} \cdot \bar{p}$ and $q_{h}>\bar{q}, p_{h}<\bar{p} ;(c)$ if $h$ has low quality and a low quality/ price ratio relative to the reference good, $q_{h} / p_{h}<\bar{q} / \bar{p}$ and $q_{h}<\bar{q}, p_{h}<\bar{p}$; and $(d)$ if $h$ is dominated by the reference good, with lower quality and higher price, $q_{h}$. $p_{h}<\bar{q} \cdot \bar{p}$ and $q_{h}<\bar{q}, p_{h}>\bar{p}$.

We are mainly interested in regime $a$, in which the decoy is located close to the other goods, that is, $\bar{q}<q_{h}$ and $\bar{p}<p_{h}$, and it is a "bad deal"; that is, it has a low quality/price ratio. In this regime the condition that $h$ has a quality/price ratio above the reference good is

$$
\frac{q_{d}}{p_{d}}<\frac{q_{h}}{p_{h}}+\frac{p_{l}}{p_{d}}\left(\frac{q_{h}}{p_{h}}-\frac{q_{l}}{p_{l}}\right) .
$$

We can write this as

$$
q_{d}<p_{d} \frac{q_{h}}{p_{h}}+p_{l}\left(\frac{q_{h}}{p_{h}}-\frac{q_{l}}{p_{l}}\right)
$$

So the upper boundary for $d$ has slope $q_{h} / p_{h}$ but is shifted downward by a factor proportional to $q_{h} / p_{h}-q_{l} / p_{l}$. In particular,

$$
\frac{q_{d}}{p_{d}}<\frac{q_{h}}{p_{h}}<\frac{q_{l}}{p_{l}} .
$$

(Both regimes $a$ and $b$ impose upper bounds on $q_{d}$. In regime $b, \bar{q}_{d}<q_{h}, \bar{p}>p_{h}$ and the condition on $q_{h} \cdot p_{h}$ yields $q_{d}<q_{h}\left(3 p_{h} / \bar{p}-1\right)-q_{l}$. Regimes $c$ and $d$ instead impose lower bounds on $q_{d}$.)

In regime $a, h$ is quality salient, which guarantees that it is preferred to $l$. To see that alternative $d$ is never chosen, two cases are distinguished: either $d$ has higher quality and a lower quality/price ratio than $h$, in which case it is price salient; or it has lower quality and a lower quality/price ratio than $h$, in which case either it can be dominated $\left(q_{d}<q_{h}\right.$ and $\left.p_{d}>p_{h}\right)$ or not. In either case, by being quality salient, $h$ is overvalued relative to $d$. Thus, a small enough $\delta$ can be found such that $h$ is chosen. A sufficient condition for $h$ to be chosen, for any $\delta$, is that the decoy lies on a lower rational indifference curve than $h$. This is guaranteed for dominated $d$ and by continuity for some $d$ with $q_{d}>q_{h}$ as well. In fact, this holds for all decoys in regime $a$.

Consider now case ii. Since $q_{l} / p_{l}<q_{h} / p_{h}$, so that good $h$ has a relatively high quality/price ratio, quality is salient in $\{l, h\}$ and $h$ is chosen. Given the constraints $\bar{q}<q_{h}$ and $\bar{p}<p_{h}$, adding a decoy $d$ to the choice set makes $h$ price salient when 
it increases the quality/price ratio of the average good to the level where $q_{h} / p_{h}$ $<\bar{q} / \bar{p}$. However, this is excluded by the condition that the decoy is a "bad deal," namely, $q_{d} / p_{d}<\max \left\{q_{l} / p_{l}, q_{h} / p_{h}\right\}$. QED

Proof of Proposition 5

Suppose that the prices of all goods in $\mathbf{C}_{\mathrm{C}}$ are shifted by a small $\gamma>0$. Then the average price in $\mathbf{C}$ shifts by $\eta \cdot \gamma$, where $\eta$ is the share of goods in $\mathbf{C}_{\mathbf{C}}$. Consider the salience of price for goods in $\mathbf{C}_{\mathrm{C}}$ that have price $p^{*}$, that is, $\sigma\left(p^{*}+\gamma, \bar{p}+\eta \gamma\right)$. Diminishing sensitivity implies that salience decreases in $\gamma$ whenever $\eta=1$ or when $\eta<1$ but $p^{*}<\bar{p}$. The reason is that in either situation the average payoff level increases but the difference between payoffs weakly decreases.

For salience to increase in $\gamma$, it is necessary that the difference in payoffs increases as well, so that the ordering property of salience may dominate over diminishing sensitivity. A necessary condition for salience to increase is thus that $\eta<1$ and $p^{*}>\bar{p}$. The precise trade-off between payoff level and payoff difference (i.e., between diminishing sensitivity and ordering) is not pinned down by the properties of salience considered in definition 1. However, assuming homogeneity of degree zero, we get that

$$
\partial_{\gamma} \sigma\left(p^{*}+\gamma, \bar{p}+\eta \gamma\right)>0 \Leftrightarrow \partial_{\gamma} \frac{p^{*}+\gamma}{\bar{p}+\eta \gamma}>0
$$

Replacing $p^{*}$ with $p_{\mathrm{C}}^{\max }$, we get the condition in the proposition. QED

\section{Proof of Proposition 6}

The average quality in $\mathbf{C}_{\text {cont }}$ is $\bar{q}=(2 / 3) \cdot q$. The average price is $\bar{p}=(1 / 3)(p$ $+\mathbb{E}[p \mid \sigma])$. Thus, the salience of quality and price of good $(q, p)$ are, respectively,

$$
\sigma\left(1, \frac{2}{3}\right), \quad \sigma\left(1, \frac{1}{3}\left(1+\frac{\mathbb{E}[p \mid \sigma]}{p}\right)\right)
$$

It follows that quality is salient when

$$
p \in\left(\mathbb{E}[p \mid \sigma] \cdot \frac{2}{7}, \mathbb{E}[p \mid \sigma]\right) \text { or } \mathbb{E}[p \mid \sigma] \in\left(p, \frac{7 p}{2}\right) .
$$

Note that as $p$ varies in this range, it can take values larger or smaller than the reference price $\bar{p}$. In turn, price is salient when

$$
\mathbb{E}[p \mid \sigma]<p \quad \text { and } \quad \mathbb{E}[p \mid \sigma]>\frac{7 p}{2} .
$$

Recall the definition of willingness to pay:

$$
\begin{array}{ll} 
& \operatorname{WTP}(q \mid(q, \mathbb{E}[p \mid \sigma]))=\sup p \\
\text { subject to } & u^{S}((q, p) \mid(q, \mathbb{E}[p \mid \sigma])) \geq u^{S}((0,0) \mid(q, \mathbb{E}[p \mid \sigma])) .
\end{array}
$$


Consider first the case in which the good is expensive relative to the reference price, $\mathbb{E}[p \mid \sigma]<p$. Then price is salient, so the consumer buys the good if and only if its discounted quality is sufficiently high, $\delta q \geq p$. Thus, WTP $=\delta q$ whenever $\mathbb{E}[p \mid \sigma]<\delta q$.

Consider now the case in which quality is salient, so the good is cheaper than the reference price, $\mathbb{E}[p \mid \sigma] \geq p$, but the price is not too low. If quality is salient, the consumer buys the good as long as its inflated quality is above its price, $q / \delta \geq p$. Thus, price can be jacked up all the way to $q / \delta$ as long as it does not change the salience ranking: $\mathrm{WTP}=\max \{q / \delta, \mathbb{E}[p \mid \sigma]\}$. As a consequence, for $\mathbb{E}[p \mid \sigma] \leq q / \delta$, WTP $=\mathbb{E}[p \mid \sigma]$. For $7 q / 2 \delta>\mathbb{E}[p \mid \sigma]>q / \delta$, we find WTP $=q / \delta$.

Finally, consider the case $\mathbb{E}[p \mid \sigma]>7 q / 2 \delta$. Now the reference price is so high that even at the highest possible price for the good, namely $q / \delta$, its price is salient. As a result, WTP goes back down to $\delta q$. QED

\section{Proof of Prediction 1}

Under the "if" step of the prediction, the consumer's choice changes from $i$ to $j$ as prices increase in parallel. This implies that $(a)$ there is a shift in the salience ranking of at least one of the goods $i$ and $j$, which benefits the valuation of $j$ relative to $i$, and (b) the shift in salience rankings is sufficient to change the consumer's choice. Because expected prices coincide with observed prices, diminishing sensitivity implies that under a price increase, any shift in salience ranking is a shift from price to quality salience. ${ }^{22}$ Since $q_{j}>q_{i}$, that is, demand for quality increases with price level, it must be that good $j$ becomes quality salient and, thus, overvalued relative to good $i$.

Turning to the "then" step of the prediction, note that because $p_{i}<\bar{p}_{0}<p_{j}$, uniformly increasing the prices of all other goods $k \neq i, j$ such that the resulting reference price equals $p_{j}$ results in good $j$ being strictly quality salient while strictly increasing the salience of price for good $i$ (thus precluding the case in which good $i$ goes from being price salient to quality salient). As a consequence, under the new price distribution, good $j$ is preferred over good $i$ so that good $i$ is no longer chosen. Yet the characteristics of goods $i$ and $j$ have not changed, and the other goods have become more expensive. This is a violation of the axiom of independence of irrelevant alternatives. QED

\section{Proof of Prediction 2}

We begin with two observations. First, as observed prices uniformly shift by $\Delta p$, the reference price shifts by $\Delta p / 2$, so that this process typically affects the salience rankings of the two goods. In particular, if the observed price of a good becomes very different from the expected price (either very low or very high), price might become salient. If instead the actual price is close to the expected price, then quality is salient. Thus, as both goods' prices increase by $\Delta p$, a given good's sa-

${ }^{22}$ Without loss of generality, we ignore the nongeneric case of equal salience of price and quality, as well as the case in which $q_{j}=\bar{q}$. The argument carries through to these cases as well. 
lience ranking follows (a subset of) the chain $P \rightarrow Q \rightarrow P$, where $P$ and $Q$ represent price and quality salience, respectively. As a consequence, the set of transitions of salience rankings of the two goods as both goods' observed prices uniformly increase are described by (a subset of) one of the following chains:

$$
\begin{aligned}
& P P \rightarrow Q P \rightarrow\{P P \text { or } Q Q\} \rightarrow P Q \rightarrow P P, \\
& P P \rightarrow P Q \rightarrow\{P P \text { or } Q Q\} \rightarrow Q P \rightarrow P P .
\end{aligned}
$$

Here the first letter denotes the salient attribute of good 1 and the second letter denotes the salient attribute of good 2. Not all possible rankings necessarily obtain. In particular, it may be impossible to have both goods be quality salient if their prices are too far apart. ${ }^{23}$

Our second observation is that the set of possible salience rankings can be ordered in terms of the difference in valuation between the lower-quality good and the high-quality good. Write $\Delta v_{X Y}$ as the difference in valuation of good 1 versus good 2 when good 1's attribute $X$ is salient and good 2's attribute $Y$ is salient:

$$
\Delta v_{X Y}= \begin{cases}\left(q_{1}-q_{2}\right)-\delta\left(p_{1}-p_{2}\right) & X Y=Q Q \\ \left(q_{1}-\delta q_{2}\right)-\left(\delta p_{1}-p_{2}\right) & X Y=Q P \\ \left(\delta q_{1}-q_{2}\right)-\left(p_{1}-\delta p_{2}\right) & X Y=P Q \\ \delta\left(q_{1}-q_{2}\right)-\left(p_{1}-p_{2}\right) & X Y=P P\end{cases}
$$

For example, the overvaluation of good 1 is larger when the salience rankings are $Q P$ than when they are $Q Q$, namely, $\Delta v_{Q P}>\Delta v_{Q Q}$. We can check that, under the quality and price rankings of goods 1 and 2, the relative valuations satisfy $\Delta v_{Q P}$ $>\Delta v_{Q Q}>\Delta v_{P P}>\Delta v_{P Q}$.

When the two observations are put together, only a certain set of shifts in demand for quality can occur under parallel price shifts. For instance, if $\Delta v_{P Q}>0$, then good 1 is chosen under any salience ranking, so that the consumer chooses good 1 at any price (we say that demand for quality is flat as a function of price levels). Similarly, if $\Delta v_{Q P}<0$, good 2 is always chosen. If $\Delta v_{P Q}<0<\Delta v_{Q P}$, then demand for quality along the entire chains (A1) and (A2) is single-peaked, namely, of the form $1 \rightarrow 2 \rightarrow 1$ or $2 \rightarrow 1 \rightarrow 2$. However, if only a subset of these chains obtain under parallel price shifts with $\Delta p \geq-p_{2}$, then demand may be monotonic (or even flat). Because salience rankings are not observable, such restrictions are not predictable ex ante. Therefore, the testable prediction is that, if two changes in preferences have been observed between $\bar{p}_{0}+\Delta p_{1}$ and $\bar{p}_{0}+\Delta p_{2}$ and between $\bar{p}_{0}+\Delta p_{2}$ and $\bar{p}_{0}+\Delta p_{3}$, then there are no other preference changes at other price levels: demand for quality is constant (and equal) for $\Delta p \in\left[-p_{2}, \Delta p_{1}\right]$ and $\Delta p>\Delta p_{3}$.

Note that if the observer has some information about the expected prices, then further predictions can be made. Suppose that the expected prices satisfy

${ }^{23}$ Moreover, the endpoints of the chain may not obtain either. For instance, as $\Delta p$ becomes large, the salience of each good's price approaches $\sigma(\Delta p, \Delta p / 2)$. If the salience function is homogeneous of degree zero, this equals a finite number, so that quality might be salient in this limit. Also, in each chain's middle step, either $P P$ or $Q Q$ obtains, but not both. 


$$
\bar{p}_{e}<p_{2}-\frac{p_{1}-p_{2}}{2}
$$

In this case, the lower-quality good costs more than the reference price $\left(\bar{p}_{e}+\bar{p}_{0}\right) / 2$, so any increase in the observed prices boosts the price salience of both goods. Moreover, since $p_{1}>p_{2}$, ordering implies that $\sigma\left(p_{1}, \bar{p}\right)>\sigma\left(p_{2}, \bar{p}\right)$, while diminishing sensitivity (together with the assumption that expected qualities coincide with observed qualities) implies that $\sigma\left(q_{1}, \bar{q}\right)<\sigma\left(q_{2}, \bar{q}\right)$. As a consequence, if the low-quality good is price salient, then so is the high-quality good. Conversely, still assuming that (A3) holds, if the high-quality good is quality salient, so is the low-quality good. These considerations restrict the possible salience rankings to the subset $Q Q \rightarrow P Q \rightarrow P P$ of chain (A1). In this case, suppose that under a price increase there is an increase in demand for quality. This means that both goods are price salient — we are in the rightmost PPstep of chain (A1) — and there are no further shifts in demand for higher price levels. Alternatively, suppose that under a price drop respecting (A3) there is an increase in demand for quality. This means that both goods are quality salient — we are in the $Q Q$ step of chain (A1) — and price cannot go lower without violating (A3). QED

\section{Proof of Proposition 7}

As in the text, consider the choice context $\mathbf{C}_{\text {sale }}$ given by $\left\{(0,0),\left(q, p_{s}\right),(q, \mathbb{E}[p])\right\}$. Consider the valuation of the good on sale, $\left(q, p_{s}\right)$. The salience of its quality is (using homogeneity of degree zero) $\sigma(q, 2 q / 3)=\sigma(1,2 / 3)$. The salience of its price is

$$
\sigma\left(p_{s}, \frac{p_{s}+\mathbb{E}[p]}{3}\right)=\sigma\left(1, \frac{1+\left(\mathbb{E}[p] / p_{s}\right)}{3}\right) .
$$

Therefore, quality is more salient than price as long as

$$
\frac{p_{f}}{p_{s}} \in\left(1, \frac{7-2 \pi}{2-2 \pi}\right)
$$

In fact, if $p_{f}$ is much higher than $p_{s}$, then the price difference among them becomes salient again. For ratios $p_{f} / p_{s}$ at which quality is salient, the willingness to pay is $p_{s}=q / \delta$, from which the result follows. QED

\section{Proof of Proposition 8}

The store can always make the high-quality good quality salient by holding a sale with a full price $p_{f h}=(4-\pi) p_{h}-2 p_{l}$ (in which case $p_{h}$ coincides with the expected quality in the choice context, $\mathbb{E}[p])$.

Instead, by holding a sale on the low-quality good, the store lowers the quality/ price ratio of the reference good. As long as $p_{f l}<p_{h}$, this makes it easier for $h$ to be quality salient as it has both higher quality and price and also a higher quality/price ratio compared to the reference good. In particular, if in the absence of 
a sale $h$ is quality salient and is chosen by the consumer, holding the sale for $l$ has no effect on the consumer's choice. QED

\section{References}

Azar, Ofer. 2007. "Relative Thinking Theory." J. Socio-economics 36 (1): 1-14.

Blattberg, Robert, Richard Briesch, and Edward J. Fox. 1995. "How Promotions Work." Marketing Sci. 14 (3): G122-G132.

Bodner, Ronit, and Drazen Prelec. 1994. "The Centroid Model of Context Dependent Choice.” Manuscript, Massachusetts Inst. Tech.

Bordalo, Pedro, Nicola Gennaioli, and Andrei Shleifer. 2012. "Salience Theory of Choice under Risk.” Q.J.E. 127 (3): 1243-85.

Chetty, Raj, Adam Looney, and Kory Kroft. 2009. "Salience and Taxation: Theory and Evidence." A.E.R. 99 (4): 1145-77.

Cunningham, Tom. 2011. "Comparisons and Choice.” Manuscript, Harvard Univ.

Eliaz, Kfir, and Ran Spiegler. 2011. "Consideration Sets and Competitive Marketing." Rev. Econ. Studies 78 (1): 235-62.

Fehr, Ernst, and Antonio Rangel. 2011. "Neuroeconomic Foundations of Economic Choice-Recent Advances." J. Econ. Perspectives 25 (4): 3-30.

Feigenson, Lisa, Stanislas Dehaene, and Elizabeth Spelke. 2004. "Core Systems of Number." Trends Cognitive Sci. 8 (7): 307-14.

Gabaix, Xavier. 2012. "A Sparsity-Based Model of Bounded Rationality, Applied to Basic Consumer and Equilibrium Theory." Working paper, New York Univ.

Gabaix, Xavier, and David Laibson. 2006. "Shrouded Attributes, Consumer Myopia, and Information Suppression in Competitive Markets." Q.J.E. 121 (2): 505-40.

Gennaioli, Nicola, and Andrei Shleifer. 2010. "What Comes to Mind." Q.J.E. 125 (4): 1399-1433.

Hare, Todd, Colin Camerer, and Antonio Rangel. 2009. "Self-Control in DecisionMaking Involves Modulation of the vmPFC Valuation System." Science 324 (5927): 646-48.

Hastings, Justine, and Jesse Shapiro. 2013. "Fungibility and Consumer Choice: Evidence from Commodity Price Shocks.” Q.J.E. 128 (4): 1449-98.

Hauser, John, and Birger Wernerfelt. 1990. "An Evaluation Cost Model of Consideration Sets." J. Consumer Res. 16 (4): 393-408.

Heath, Timothy, and Subimal Chatterjee. 1995. "Asymmetric Decoy Effects on Lower-Quality versus Higher-Quality Brands: Meta-Analytic and Experimental Evidence." J. Consumer Res. 22 (3): 268-84.

Heidhues, Paul, and Botond Koszegi. 2008. "Competition and Price Variation When Consumers Are Loss Averse." A.E.R. 98 (4): 1245-68.

Huber, Joel, John Payne, and Christopher Puto. 1982. "Adding Asymmetrically Dominated Alternatives: Violations of the Regularity and Similarity Hypothesis." J. Consumer Res. 9 (1): 90-98.

Jahedi, Salar. 2011. "A Taste for Bargains.” Manuscript, Univ. Arkansas.

Kahneman, Daniel. 2011. Thinking, Fast and Slow. New York: Farrar, Straus \& Giroux.

Kahneman, Daniel, and Dale Miller. 1986. "Norm Theory: Comparing Reality to Its Alternatives.” Psychological Rev. 93 (2): 136-53.

Kahneman, Daniel, and Amos Tversky. 1979. "Prospect Theory: An Analysis of Decision under Risk.” Econometrica 47 (2): 263-92.

—. 1984. "Choices, Values and Frames." American Psychologist 39:341-50. 
Kamenica, Emir. 2008. "Contextual Inference in Markets: On the Informational Content of Product Lines.” A.E.R. 98 (5): 2127-49.

Kim, Jongmin, Nathan Novemsky, and Ravi Dhar. 2012. "Adding Small Differences Can Increase Similarity and Choice.” Psychological Sci. 24 (2): 225-29.

Koszegi, Botond, and Matthew Rabin. 2006. "A Model of Reference-Dependent Preferences." Q.J.E. 121 (4): 1133-65.

Koszegi, Botond, and Adam Szeidl. 2013. "A Model of Focusing in Economic Choice." Q.J.E. 128 (1): 53-104.

Lazear, Edward P. 1986. "Retail Pricing and Clearance Sales." A.E.R. 76 (1): 14-32.

Mullainathan, Sendhil. 2002. "Thinking through Categories." Manuscript, Harvard Univ.

Ortmeyer, Gwen, John Quelch, and Walter Salmon. 1991. "Restoring Credibility to Retail Pricing." Sloan Management Rev. 33 (1): 55-66.

Roberts, John, and James Lattin. 1997. "Consideration: Review of Research and Prospects for Future Insights.” J. Marketing Res. 34 (3): 406-10.

Savage, Daniel. 1954. The Foundation of Statistics. New York: Dover

Schwartzstein, Joshua. 2012. "Selective Attention and Learning." Manuscript, Harvard Univ.

Simonson, Itamar. 1989. "Choice Based on Reasons: The Case of the Attraction and Compromise Effects." J. Consumer Res. 16 (2): 158-74.

Simonson, Itamar, and Amos Tversky. 1992. "Choice in Context: Tradeoff Contrast and Extremeness Aversion." J. Marketing Res. 29 (3): 281-95.

Sobel, Joel. 1984. "The Timing of Sales." Rev. Econ. Studies 51 (3): 353-68.

Taylor, Shelley, and Suzanne Thompson. 1982. "Stalking the Elusive Vividness Effect.” Psychological Rev. 89 (2): 155-81.

Thaler, Richard. 1980. "Toward a Positive Theory of Consumer Choice." J. Econ. Behavior and Org. 1 (1): 39-60.

- 1985. "Mental Accounting and Consumer Choice." Marketing Sci. 4 (3): 199-214. 206.

. 1999. "Mental Accounting Matters.” J. Behavioral Decision Making 12:183-

Tversky,

sky, Amos. 1972. "Choice by Elimination." J. Math. Psychology 9 (4): 341-67.

Tversky, Amos, and Daniel Kahneman. 1991. "Loss Aversion in Riskless Choice: A Reference-Dependent Model.” Q.J.E. 106 (4): 1039-61.

Tversky, Amos, and Itamar Simonson. 1993. "Context Dependent Choices." Marketing Sci. 39 (10): 1179-89.

Varian, Hal. 1980. "A Model of Sales." A.E.R. 70:651-59.

Wernerfelt, Birger. 1995. "A Rational Reconstruction of the Compromise Effect: Using Market Data to Infer Utilities.” J. Consumer Res. 21 (4): 627-33.

Woodford, Michael. 2012. "Inattentive Valuation and Reference-Dependent Choice." Manuscript, Columbia Univ. 